\title{
Multi-scale Observation and Data-driven Modeling of Magnetic Reconnection in Flaring Chromospheric Plasmas on the Sun
}

\section{Xiaoli Yan ( $\nabla$ yanxl@ynao.ac.cn )}

Yunnan Observatories,CAS

\section{Zhike Xue}

Yunnan Observatories

Chaowei Jiang

Harbin Institute of Technology, Shenzhen https://orcid.org/0000-0002-7018-6862

\section{Eric Priest}

School of Mathematics and Statistics

\section{Bernhard Kliem}

University of Potsdam https://orcid.org/0000-0002-5740-8803

\section{Liheng Yang}

Yunnan Observatories

Jincheng Wang

Yunnan Observatories CAS

\section{Defang Kong}

Yunnan Observatories

\section{Yongliang Song}

Key Laboratory of Solar Activity, National Astronomical Observatories, Chinese Academy of Sciences

\section{Xueshang Feng}

Chinese academy of sciences

\section{Zhong Liu}

Yunnan Observatories, Chinese Academy of Sciences, China

\section{Article}

Keywords: flaring chromospheric plasmas, planetary physics, solar flares

Posted Date: February 19th, 2021

DOl: https://doi.org/10.21203/rs.3.rs-232633/v1 
License: (c) (i) This work is licensed under a Creative Commons Attribution 4.0 International License. Read Full License

Version of Record: A version of this preprint was published at Nature Communications on February 2nd, 2022. See the published version at https://doi.org/10.1038/s41467-022-28269-w. 


\title{
Multi-scale Observation and Data-driven Modeling of Magnetic Reconnection in Flaring Chromospheric Plasmas on the Sun
}

\author{
Xiaoli Yan ${ }^{1,2,3}$, Zhike Xue ${ }^{1,3}$, Chaowei Jiang ${ }^{4}$, E. R. Priest ${ }^{5}$, \\ Bernhard Kliem $^{6}$, Liheng Yang ${ }^{1,3}$, Jincheng Wang ${ }^{1,3}$, Defang Kong ${ }^{1,3}$, \\ Yongliang Song ${ }^{7}$, Xueshang Feng ${ }^{2}$, Zhong Liu ${ }^{1,8}$
}

February 11, 2021

Magnetic reconnection is a multi-faceted process of energy conversion in astrophysical, space and laboratory plasmas that operates at microscopic scales but has macroscopic drivers and consequences. Solar flares present a key laboratory for its study, leaving imprints of the microscopic physics in radiation spectra and allowing the macroscopic evolution to be imaged, yet a full observational characterization remains elusive. Here we combine high resolution imaging and spectral observations of a solar flare at multiple wavelengths with data-driven magnetohydrodynamic modeling to study the dynamics of the involved plasma from the current sheet to the plasmoid scale. The flare resulted from the interaction of a twisted filament and chromospheric fibrils. By inferring the reconnection to be fast and mediated by plasmoids, the relevance of this reconnection mode is found to extend beyond hot flare plasmas to such cool structures in the chromosphere, which have many analogs in astrophysical objects.

${ }^{1}$ Yunnan Observatories, Chinese Academy of Sciences, Kunming, Yunnan 650216, China.(email: yanxl@ynao.ac.cn)

${ }^{2}$ State Key Laboratory of Space Weather, Chinese Academy of Sciences, Beijing 100190, China.

${ }^{3}$ Center for Astronomical Mega-Science, Chinese Academy of Sciences, 20A Datun Road, Chaoyang District, Beijing, 100012, China. 
${ }^{4}$ Institute of Space Science and Applied Technology, Harbin Institute of Technology, Shenzhen, 5180055, China

${ }^{5}$ Mathematics Institute, University of St Andrews, St Andrews, KY16 9SS, UK.

${ }^{6}$ Institute of Physics and Astronomy, University of Potsdam, Potsdam 14476, Germany.

${ }^{7}$ Key Laboratory of Solar Activity, National Astronomical Observatories, Chinese Academy of Sciences, Beijing 100012, China.

${ }^{8}$ University of Chinese Academy of Sciences, Yuquan Road, Shijingshan Block, Beijing 100049, Peoples Republic of China.

\section{Introduction}

Solar flares result from the most dramatic events of energy release by magnetic reconnection on the Sun and provide a broad range of observational detail about the involved processes ${ }^{[1-3]}$. In the standard flare model for eruptive events ${ }^{[4-7]}$, magnetic reconnection proceeds in a vertical current sheet spawned on the underside of an erupting flux rope which also drives a coronal mass ejection (CME). Rising arcades of reconnected hot, initially cuspshaped magnetic loops ("flare loops"), rooted in a pair of bright "flare ribbons", are formed in the downward reconnection outflow. Confined flares result when the erupting flux is stopped by overlying flux or when neighboring flux system are pressed against each other, and a reconnecting current sheet is formed in the interface ${ }^{[8-10]}$. A filament extending along the polarity inversion line of the source region often traces the activated flux. These large-scale geometries of the reconnecting current sheet and its drivers and consequences are well established ${ }^{[1-20]}$.

High resolution imaging observations provide rich information about details of the reconnection process which result from its internal dynamics, especially the formation of plasmoids ${ }^{[21,22]}$ and turbulent structures ${ }^{[23]}$, and from the complexity and three-dimensional (3D) nature of the solar magnetic field $^{[24,25]}$. Spectral and multi-wavelength data reveal aspects of the microscopic processes, e.g., particle acceleration ${ }^{[26,27]}$, the nonthermal-thermal energy partition ${ }^{[28]}$, the plasmoid instability ${ }^{[29,30]}$, and bursty ${ }^{[31]}$ or turbulent reconnection ${ }^{[32,33]}$, in addition to flows ${ }^{[34]}$. Finally, besides hot flare plasmas, the Sun also allows the reconnection of cool chromospheric structures to be studied ${ }^{[24,35]}$. However, despite this broad range of information, comprehensive simultaneous imaging and spectral observations are still limited. 
In particular, while individual components of the reconnection in- and outflows have often been observed ${ }^{[15,18,21,34,36,37]}$, we are aware of only two events revealing all four flows ${ }^{[19,35]}$ but these have no or only limited spectral information about the involved plasma. Such limitation prevents the mode of reconnection, i.e. Petschek-like ${ }^{[12]}$, plasmoid-mediated ${ }^{[29]}$, collisionless ${ }^{[38]}$, or turbulent ${ }^{[39]}$, to be inferred.

Here we present multi-wavelength imaging and spectral evidence for magnetic reconnection in a strong (M-class) confined solar flare, traced by the cool plasma of the interacting filament and chromospheric fibrils and driven by emerging flux. The flare is unique in that it displayed all four reconnection flows, plasmoids not only in the current sheet but also in the separatrices, was fully covered by spectroscopic observations, and yielded the first hard Xray images from a current sheet on the solar disk. This allows us to measure both inflows and both outflows and to infer the mode of reconnection from the analysis of the physical conditions in the current sheet. We also perform a state-of-the-art, data-driven numerical simulation of the reconnection event, which supports our interpretation of the observational data.

\section{Results}

High-resolution imaging of the reconnection flows. The confined M2.2 flare occurred in Active Region NOAA 11967 at S13E04 on 2014 February 2, starting at 07:17 UT, peaking at 08:20 UT, and ending at 08:29 UT. This was a large active region with a $\beta \gamma \delta$ sunspot configuration. Several of its main flux components were already decaying, but there was also new flux emerging into the region. Driven by these changes, many C- and M-class flares were produced during its passage across the solar disk ${ }^{[40,41]}$.

Figure 1a shows an $\mathrm{H} \alpha$ image from the New Vacuum Solar Telescope (NVST) ${ }^{[42]}$ superimposed with magnetic flux contours from the Solar Dynamics Observatory (SDO)/Helioseismic and Magnetic Imager (HMI) ${ }^{[43]}$ magnetogram in Fig. 1b. The red curved lines in Fig. 1b indicate the positions of the reconnecting structures: chromospheric fibrils on the east (left) side and a filament, which was contained in a twisted flux rope ${ }^{[44]}$, on the west (right) side. The evolution of the flare as seen by the NVST in $\mathrm{H} \alpha$ is displayed in Fig. 1c-h and the related Supplementary Movie 1. Here the white arrows indicate the reconnecting structures at 08:04:54 UT. The structures approached each other and began to reconnect. What may well be a current sheet can be seen at the junction of the two structures marked by the blue arrows in Fig. 1d-f. The newly formed threads are indicated by black arrows 
in Fig. 1d,e. Both sets of new, reconnected structures displayed the cusp characteristic of newly reconnected field lines, as outlined by the dashed blue curved lines in Fig. 1f,g. Simultaneously with the magnetic reconnection, the M-class flare occurred (see the GOES soft X-ray light curve in Fig. 2a and the Reuven Ramaty High-Energy Solar Spectroscopic Imager (RHESSI) ${ }^{[45]}$ X-ray counts in the 3-6, 6-12, 12-25, and 25-50 keV bands in Fig. 2b).

The projected in- and outflow velocities were measured using time-slices of the intensity along the artificial slits A-B and C-D shown by the red and blue lines, respectively, in Fig. 1h. Up to 08:14 UT, the inflows are found to be $v_{\mathrm{i}}=0.06 \mathrm{~km} \mathrm{~s}^{-1}$ on the east side and $v_{\mathrm{i}}=0.05 \mathrm{~km} \mathrm{~s}^{-1}$ on the west side; see the dashed red lines in Fig. 2c. The outflows are measured from $v_{\mathrm{o}}=2.0$ to $3.7 \mathrm{~km} \mathrm{~s}^{-1}$ in southward direction, which is presumably downward, and $v_{\mathrm{o}}=$ 3.4 to $3.8 \mathrm{~km} \mathrm{~s}^{-1}$ in northward direction, which is presumably upward; see the dashed blue lines in Fig. 2d. The resulting reconnection rate, $M=v_{\mathrm{i}} / v_{\mathrm{o}}=$ 0.01-0.03, clearly indicates a fast reconnection process. We also measure the outflows along the cusp lines (magnetic separatrices) attached to the ends of the current sheet (see the curved lines E-F and G-H in Fig. 1h). These are found to lie in the ranges $2.3-5.5 \mathrm{~km} \mathrm{~s}^{-1}$ and $0.8-2.6 \mathrm{~km} \mathrm{~s}^{-1}$ for the south and north cusp lines, respectively (see Fig. 2d,e). As the reconnection continues, an unusual feature of the event is that the chromospheric fibrils and the main body of the filament began to separate from the reconnection region after 08:14 UT with velocities of 0.98 and $0.39 \mathrm{~km} \mathrm{~s}^{-1}$ on the east and west sides, respectively (see Fig. 2b), whereas some of the lower chromospheric fibrils and threads of the filament still participated in the reconnection process. This is considered further in the Discussion.

EUV spectroscopy of reconnection flows and turbulence. The Hinode/EUV Imaging Spectrometer (EIS) ${ }^{[46]}$ observations covered essentially the whole reconnection event. Figure 3 shows the intensity $(\mathrm{a}-\mathrm{c})$, Doppler velocity $(\mathrm{d}-\mathrm{f})$, and non-thermal velocity $(\mathrm{g}-\mathrm{i})$. There is a strong red shift in the southern cusp-shaped structure, with the maximum Doppler velocity reaching about $80 \mathrm{~km} \mathrm{~s}^{-1}$. The blue shift at the northern cusp reaches about $40 \mathrm{~km} \mathrm{~s}^{-1}$. However, the true peak velocity may be higher at the north side (as also indicated by the projected velocities), because the selected spectral line obviously samples only part of the upward reconnection outflow, due to a reduction of density and temperature (adiabatic cooling) in the free upward expansion of the plasma. Nevertheless, both reconnection outflows are spectroscopically detected and prove to be significantly higher near the edges of the current sheet than the outflows inferred from the $\mathrm{H} \alpha$ structures 
further away from the current sheet (Figs. 1h and 2d). It is also clear that the outflow is mainly downward at the southern edge of the current sheet and mainly upward at the northern edge. The implied reconnection rate, $M \sim 0.0007-0.024$ for the southern outflow, is still fast. The cusp-shaped structures also appear in the non-thermal velocity maps. The highest nonthermal velocities were located in the current sheet, especially in the tip of the cusp-shaped structures, with values in the range $\sim 180-300 \mathrm{~km} \mathrm{~s}^{-1}$, which significantly exceed the projected and Doppler velocities of the reconnection outflows. This suggests that the reconnection proceeds in a highly dynamic manner, with plasmoid-mediated reconnection ${ }^{[47,48]}$ being the obvious candidate for the specific mode of reconnection realized in the event. To determine the electron density in the current sheet, we used the theoretical line intensity ratios of the Fe XIV $(\lambda 264.92 / \lambda 274.37)$ density sensitive pair, which were calculated by using the CHIANTI atomic package ${ }^{[49][50]}$. Although the line $\lambda 274.37$ is blended with Si VII $\lambda 274.18$, the blend can be safely ignored due to its low intensity compared with $\lambda 274.37$ in active region conditions. The number density values in the current sheet are found to scatter in the range from $10^{8}$ to $10^{12} \mathrm{~cm}^{-3}$, similar to typical densities in filaments ${ }^{[51]}$. The highest values are located in the southern (lower) cusp.

Plasmoids. During the early, slow-rise phase of the flare, from 07:57 to 08:06 UT, many small bright blobs were observed, moving from the current sheet to the legs of the cusp-shaped structures (see Supplementary Movie 1). Figure 4 and and the related Supplementary Movie 2 show the highlighted fine structures of the SDO/AIA $211 \AA$ images revealed by the unsharp masking technique. There are many bright blobs marked by the red arrows moving from the reconnection region (see Fig. 4a-f). The velocities of the blobs along the reconnected field lines are from $79 \mathrm{~km} \mathrm{~s}^{-1}$ to $208 \mathrm{~km} \mathrm{~s}^{-1}$ (see the time-distance diagram, Fig. 4g, obtained along the green-dotted line in Fig. 4a). To the best of our knowledge, the phenomenon that blobs move from the reconnection region along the separatrices, as found in a 3D particle simulation of reconnection ${ }^{[52]}$, is observed here for the first time. Their number, more than ten, is among the highest reported so far for a single reconnection event ${ }^{[21,53]}$. They support the interpretation of the high Fe XVII line widths as an indication of plasmoid-mediated reconnection. The increasing brightness and source width of the emissions prevented the detection of blobs by unsharp masking at later times, but their continued formation is suggested by the continued presence of high nonthermal velocities in the EIS spectra, efficient particle acceleration revealed by the hard X-rays, and the 
numerical modeling below.

Heating. The heating and brightening resulting from the reconnection can also clearly be seen in all extreme ultraviolet (EUV) and UV wavelengths of the SDO/Atmospheric Imaging Assembly (AIA) ${ }^{[54]}$. The Supplementary Movie 3 shows the evolution of the EUV and UV observations at eight wavelengths and of the line-of-sight magnetogram. The cusp-shaped regions of outflowing hot plasma are obvious, as is the current sheet between them.

Six EUV wavelengths observed by AIA were used to obtain the differential emission measure (DEM), total EM, and temperature maps shown in Fig. 5a$\mathrm{f}$ and Supplementary Movie 4. The X-shaped geometry of the reconnection region can be seen in the whole temperature range of $T>1 \mathrm{MK}$, i.e., the cool plasma of the interacting filament and chromospheric fibrils is heated to coronal and flare temperatures. The peak temperature in the reconnection region reaches about $10 \mathrm{MK}$.

X-ray spectroscopy of accelerated particles. The X-ray sources from RHESSI in Fig. $5 \mathrm{~g}-\mathrm{i}$ show that $\mathrm{X}$-ray emission $>3 \mathrm{keV}$ is emitted from the beginning of the event's slow-rise phase (Fig. 5g). Higher energies are reached following the development of the flare, and the highest energy release rate into hard X-rays around 08:18 UT coincides with the steepest slope of the soft X-ray light curve (Fig. 2a), as is typically the case in flares. The emission comes mainly from the current sheet, different from eruptive flares, where the foot point sources are usually strongest. Thus, the hard X-rays provide a direct diagnostic of the conditions in the current sheet. These are the first maps of hard X-ray emission from a reconnecting current sheet in a confined flare on disk. The emission originates preferentially from the lower (southern) cusp, where the density in the sheet was found to be highest, similar to limb flares with occulted foot points ${ }^{[55]}$.

Figure 6 shows the RHESSI hard X-ray photon spectra taken at about 08:12 UT and 08:19 UT. The temperatures derived from the fitted spectra are 13.1 MK (Fig. 6a) and 33.6 MK (Fig. 6b); this extends the results of the DEM analysis of the AIA EUV data which are limited to $\approx 11 \mathrm{MK}$. Moreover, the spectra show both thermal and non-thermal components. The emission above $\sim 25 \mathrm{keV}$ is of non-thermal nature, indicating particle acceleration in the current sheet by the fast reconnection process. Particle acceleration by reconnection is known to be particularly efficient when multiple plasmoids form, implying multiple X-lines ${ }^{[27,56,57]}$. This corroborates the deduction above that plasmoid-mediated reconnection was at work.

Magnetic field structure. Using the nonlinear force free field (NLFFF) 
extrapolation of the photospheric vector magnetogram from SDO/HMI at 07:36 UT (see Methods), the magnetic structure of the filament and the involved magnetic loops prior to the onset of reconnection are obtained. Figure 7 shows the magnetic structure of the filament, the magnetic loops, and the map and iso-surface of the magnetic squashing degree $\mathrm{Q}^{[58]}$, which measures the gradient in the field line foot point mappping. High values of $\mathrm{Q}$, known as quasi-separatrix layers (QSLs), are the preferred locations for the formation of current sheets and the onset of reconnection ${ }^{[59]}$. As can be seen in the NLFFF extrapolation, the magnetic field lines from the main positive sunspot in the east part of the active region connect to the diffuse negative polarity in the north (the pink lines), while the field lines of the filament from the smaller positive spot in the west connect to the main negative spot (the green lines). The latter, weakly twisted magnetic structure (Fig. 7a) is in good agreement with the shape of the filament in the $\mathrm{H} \alpha$ center observation (Fig. 1a). Figures $7 \mathrm{~b}-\mathrm{c}$ show the map of $\log Q$ in the reconnection region at heights $Z=0$ and $Z=2.9 \mathrm{Mm}$ above the photosphere. The photospheric magnetogram and the iso-surface $\log Q=4$ in Fig. $7 \mathrm{~d}$ reveal the X-shaped structure of high $\log Q$ values that facilitates the onset of reconnection at the observed position. The vertical cross-section of $\log Q$ in Fig. 7e demonstrates that the separatrices run between the flux bundles, at the position where the current sheet forms.

MHD simulation. We further simulated the reconnection process using a data-driven high-resolution magnetohydrodynamic (MHD) model. Since this realistic, data-driven model of the current sheet includes a guide field component in the direction of the current flow, the plasmoids seen in the current density distribution (Fig. 8e-f) are small, three-dimensional flux ropes in the field line plots ${ }^{[52]}$ (Fig. 8a-d). As observed in two-dimensional simulations ${ }^{[47,48]}$, as well as in the observations presented above, plasmoids form dynamically and repeatedly, and are accelerated along the current sheet. Two new plasmoids are seen to form in Fig. 8f. Between the plasmoids, the current sheet steepens to the limit allowed by the numerical resolution. The simulation obviously corresponds well to the observations from the global to the plasmoid scale. The plasmoids appeared only after adaptive mesh refinemnt was applied to permit the current sheet width to shrink to an aspect ratio of $\sim 0.01$, in agreement with the theoretical prediction for the occurrence of the plasmoid instability ${ }^{[60]}$.

Released energy. A rough estimate of the way reconnection releases the magnetic energy required to power an M-class flare $\left(\sim 10^{24} \mathrm{~J}\right)$ can be made 
as follows. We expect that part of the energy is released in the reconnecting current sheet and a further part is released by the untwisting of the filamentflux rope after it is connected to the diffuse remote polarity. Suppose first that oppositely directed magnetic field of strength $B_{\mathrm{i}}$ is brought in from both sides at speed $v_{\mathrm{i}}$ for a time $\tau$ towards a reconnection region of surface area $L_{0}{ }^{2}$, where the current sheet and attached slow-mode shock waves convert the energy. The magnetic energy is brought in from both sides from a volume $2 L_{0} L_{\mathrm{i}}$, where $L_{\mathrm{i}}=v_{\mathrm{i}} \tau$, and so the total energy calculated is

$$
W=\frac{B_{\mathrm{i}}^{2}}{2 \mu_{0}} L_{0}^{2} v_{\mathrm{i}} \tau .
$$

This may be evaluated for the M-class flare, where $B_{\mathrm{i}} \sim 500 \mathrm{G}$ from the extrapolation of the magnetogram which is used as the initial condition for the simulation (the average value in the reconnection region), and the values $L_{0}=15^{\prime \prime}(10.9 \mathrm{Mm}), v_{\mathrm{i}}=2 \mathrm{~km} \mathrm{~s}^{-1}$, and $\tau=70 \mathrm{~min}$ are obtained from the $\mathrm{H} \alpha$ data, to give an energy of $\sim 10^{24} \mathrm{~J}$, as required. This corresponds well to the energy release of $\sim 10^{24} \mathrm{~J}\left(9.6 \times 10^{30} \mathrm{erg}\right)$ in the simulation and agrees with the canonical picture that the flare is powered by magnetic reconnection.

Additional magnetic energy may be liberated by the untwisting of the flux rope which holds the filament. The energy associated with the twist component of the flux rope field, $B_{\phi}=(R / a) B_{0 \phi}$, can be written as

$$
W_{\mathrm{FR}}=\frac{B_{0 \phi^{2}}}{2 \mu_{0}} \pi a^{2} L_{\mathrm{R}},
$$

where $R, a$ and $L_{\mathrm{R}}$ are the rope's major and minor radii and length. Suppose the twist on the edge of the flux rope is $\Phi$, then $\Phi=\left(\mathrm{L} B_{0 \phi}\right) /\left(\mathrm{a} B_{z}\right)$ and $B_{0 \phi}$ is $\mathrm{a} \Phi B_{z} / L_{\mathrm{R}}$. In the present case, we estimate $B_{z} \sim 500 \mathrm{G}, L_{\mathrm{R}}=100^{\prime \prime}, a=5^{\prime \prime}$ and a twist of $\Phi \sim 4 \pi$, so $W_{\mathrm{FR}}=3 \times 10^{23} \mathrm{~J}$. This is significantly smaller than the energy released in the reconnecting current sheet, unless the flux tube radius is a factor of $\sim 2$ larger than we have estimated from the $\mathrm{H} \alpha$ images of the filament.

In summary, the presented high-resolution imaging and spectral observations of macroscopic flows demonstrate that fast magnetic reconnection of the field in structures originally at chromospheric temperatures released the magnetic energy which powered the solar flare. Plasmoid-mediated reconnection is strongly indicated by direct imaging and nonthermal EUV and hard Xray spectra. This is further supported by a 3D data-driven MHD simulation 
which reproduces the observed change in connectivity, agrees with estimates of the energy release, and reveals the appearance of twisted plasmoids (mini flux ropes) at the theoretically expected threshold.

\section{Discussion}

In order to understand why reconnection occurred so many times in this place, the evolution of the magnetic and flow fields in the photosphere was investigated using HMI vector magnetograms. The vector magnetogram is shown in Fig. 9a-c, where the arrows represent the horizontal magnetic field. The corresponding flow field was calculated from the radial magnetic field using the DAVE algorithm ${ }^{[61]}$ (see Methods); see Fig. 9d-f and the related Supplementary Movie 5. This reveals a flow toward the magnetic reconnection site from the west side, which pushes together oppositely directed flux in the higher atmosphere (as found in the MHD simulation). This persistent flow in the photosphere is likely to be the main reason why so many flares occurred in the same place. Additional distortions of the coronal field configuration, also likely to support the onset of reconnection, are produced by the emergence of flux very near the reconnection site (green ellipses in Fig. 9b,c) and subsequent motion toward the reconnection site. The flows at the east side are more variable, with directions along and away from the current sheet alternating. The latter can explain the separation of the reconnecting flux after $\sim 08: 14$ UT in the present event, as well as the termination of the other homologous events on the same day.

A neighboring eruption may have had an additional influence. This active region produced three M-class flares and several C-class flares in the first half of 2014 February 2. All these flares were located at the same place and had a similar morphology. The M-class flare studied here was preceded by a weaker, C-class flare above another polarity inversion line in the complex active region, lying to the east of the main positive sunspot, at $(x, y) \approx(-340,-50)$. The C-class flare was caused by the rise of flux in the corona above the filament located at that inversion line. The associated large-scale change of the coronal field, due to the expansion of that flux, may have played an additional role in pushing the chromospheric fibrils east of the reconnection region toward the filament on the west side of the region. With the relaxation of the overlying coronal field during the decay of the C-class flare (07:17-07:58 UT) ${ }^{[62]}$, the fibrils may have experienced a restoring, oppositely directed force, dragging them apart from the formed current sheet and terminating the reconnection inflow. This might have additionally 
contributed to the termination of the reconnection event.

Once the reconnection in- and outflows are established as inferred from the $\mathrm{H} \alpha$ data, reconnection would not easily cease by itself, because the process involves a positive feedback: the acceleration of the outflows drives continued inflows which transports new flux with an oppositely directed component toward the sheet. This is consistent with the MHD simulation. The fact that the reconnection event produced an M2.2 flare, more intense by a factor two than the preceding C8.9 flare, shows that the active region had stored free magnetic energy in the area of the reconnection event. This free energy was carried by the electric current associated with the antiparallel field component of the reconnecting structures. Since these structures still existed after the flare, the alternative possibility for the relatively quick ending of the reconnection event by a depletion of the available free magnetic energy appears less likely than the action of an external driver.

\section{Methods}

Observational data Images in the center of the $\mathrm{H} \alpha$ line at $6562.8 \AA$ obtained at the $1 \mathrm{~m}$ New Vacuum Solar Telescope (NVST) at Fuxian Lake ${ }^{[42]}$, are used in this study. They have a pixel size of $0 .{ }^{\prime \prime} 163$ and a cadence of $12 \mathrm{~s}$. The data are calibrated from Level 0 to Level 1 with dark current subtracted and flat field corrected, and then the calibrated images are reconstructed to Level $1+$ using a speckle masking method ${ }^{[63]}$.

Full-disk UV and EUV images observed by SDO/AIA ${ }^{[54]}$ are also used to clarify the process of magnetic reconnection. These images have a $12-\mathrm{s}$ cadence and a spatial resolution of $0 .^{\prime \prime} 6$ per pixel. Vector magnetograms from the Space Weather HMI Active Region Patch (SHARP) series observed by the Helioseismic and Magnetic Imager (HMI) ${ }^{[43,64,65]}$ on board SDO are used. These allow us to infer the photospheric velocity field and to perform a datadriven simulation of the event. The vector magnetograms have a pixel scale of about $0 .{ }^{\prime \prime} 5$ and a cadence of 12 minutes. They are derived by using the Very Fast Inversion of the Stokes Vector algorithm ${ }^{[66]}$. The minimum energy $\operatorname{method}^{[67,68]}$ is used to resolve their 180 degree azimuthal ambiguity. The images are remapped using a Lambert (cylindrical equal area) projection centered on the midpoint of the AR, which is tracked at the Carrington rotation rate ${ }^{[69]}$.

The spectral data observed by the EUV Imaging Spectrometer (EIS) on board the Hinode mission were used to derive the Doppler velocity and nonthermal velocity in the reconnection region. Hinode/EIS ${ }^{[46]}$ is a scanning slit 
spectrometer that uses two wave bands in the EUV: $170-210 \AA$ and 250 $290 \AA$. The spectral resolution is about $0.0223 \AA$ pixel $^{-1}$. It can measure velocities at an accuracy of a few $\mathrm{km} \mathrm{s}^{-1}$. Fortunately, the EIS observation covered the whole duration of the reconnection event and nearly its whole area. It thus provides an excellent opportunity to conduct a spectroscopic study of magnetic reconnection. We use the line Fe XVII $255 \AA(\log T=6.6)$. The field of view (FOV) is $161^{\prime \prime}$ in the raster direction and $152^{\prime \prime}$ in the slit direction. The spectral profiles are fitted with single Gaussian functions. The reference wavelength used to determine the Doppler and non-thermal velocities is taken from the average of each raster. The sparse raster used the $2^{\prime \prime}$ slit with a step size of $2 .^{\prime \prime} 995$ and an exposure time of 4-6 s.

Data from RHESSI ${ }^{[45]}$ are also employed to study the soft and hard X-ray sources during the M-class flare. The energy ranges of 3-6, 6-12, 12-25, and 25-50 keV were selected to observe the evolution of these sources during the magnetic reconnection. The accumulation time is $30 \mathrm{~s}$ for constructing X-ray images by using the Clean imaging algorithm.

Data-driven simulation. The full set of time-dependent 3D MHD equations were solved, imposing bottom boundary conditions driven continuously ${ }^{[70,71]}$ by the changing photospheric vector magnetic field observed by SDO/HMI (see Observational data). The background plasma is initially in a hydrostatic, isothermal state with $T=10^{6} \mathrm{~K}$ (sound speed $c_{S}=128 \mathrm{~km} \mathrm{~s}^{-1}$ ) in solar gravity. Its density is configured to make the plasma $\beta$ as small as $2 \times 10^{-3}$ (the maximum Alfvén velocity $v_{\mathrm{A}}$ is $4 \mathrm{Mm} \mathrm{s}^{-1}$ ) to mimic the coronal low- $\beta$ and highly tenuous conditions. The plasma thermodynamics are simplified to be adiabatic. The bottom boundary of the model is assumed to be the coronal base, and also the magnetic field measured on the photosphere is used as a reasonable approximation to the field at the coronal base. To obtain the detailed structure in the current sheet, an adaptive refinement mesh is used with highest resolution of $1 / 16$ arcsecond (i.e., $45 \mathrm{~km}$ ) to capture the fine structures in the reconnecting current sheet.

Unsharp masking technique. The unsharp masking technique is applied to SDO/AIA $211 \AA$ images to highlight fine structures like blobs. First, a background image by smoothing the original image is obtained as an unsharp masked image. Second, the enhanced image is the residual of the original image subtracting the background one. A smoothing window of $7 \times 7$ pixels is adopted for optimal effects. 
NLFFF extrapolation. The three-dimensional magnetic structures of the filament and the magnetic loops are reconstructed by using an optimization algorithm $^{[72]}$. The bottom vector magnetic field data observed by SDO/HMI are modified to remove most of the net force and torque that usually results in an inconsistency between the photospheric magnetic field and the force-free assumption in the NLFFF models by using a preprocessing procedure $^{[73]}$. The visualization of the 3D magnetic field is realized by the software Paraview.

RHESSI spectrum. RHESSI hard X-ray photon spectra were created by the Object Spectral Executive (OSPEX) ${ }^{[74]}$ software with the background subtracted. A single-temperature thermal bremsstrahlung radiation function ("vth") and the thick-target non-thermal bremsstrahlung with an isotropic pitch-angle distribution ("thick2") were adopted to fit the spectra.

Velocity field calculation. The differential affine velocity estimator (DAVE) ${ }^{[61,75]}$ has been applied to the HMI vector magnetograms to derive the photospheric flow field. The DAVE method combines the advection equation and a differential feature tracking technique to detect flows. We used a window size of 19 pixels, which is large enough to include structure information and small enough to have a good spatial resolution.

\section{References}

[1] Priest, E. R. \& Forbes, T. G. The magnetic nature of solar flares. Astron. Astrophys. Rev. 10, 313-377 (2002).

[2] Fletcher, L. et al. An Observational Overview of Solar Flares. Space Sci. Rev. 159, 19-106 (2011). 1109.5932.

[3] Benz, A. O. Flare Observations. Living Reviews in Solar Physics 14, 2 (2017).

[4] Carmichael, H. A Process for Flares. NASA Special Publication 50, 451 (1964).

[5] Sturrock, P. A. Model of the High-Energy Phase of Solar Flares. Nature 211, 695-697 (1966). 
[6] Hirayama, T. Theoretical Model of Flares and Prominences. I: Evaporating Flare Model. Solar Phys. 34, 323-338 (1974).

[7] Kopp, R. A. \& Pneuman, G. W. Magnetic reconnection in the corona and the loop prominence phenomenon. Solar Phys. 50, 85-98 (1976).

[8] Ji, H., Wang, H., Schmahl, E. J., Moon, Y. J. \& Jiang, Y. Observations of the Failed Eruption of a Filament. Astrophys. J. Lett. 595, L135L138 (2003).

[9] Török, T. \& Kliem, B. Confined and Ejective Eruptions of Kinkunstable Flux Ropes. Astrophys. J. Lett. 630, L97-L100 (2005). astro-ph/0507662.

[10] Li, L. et al. Magnetic reconnection between a solar filament and nearby coronal loops. Nature Physics 12, 847-851 (2016). 1605.03320.

[11] Tsuneta, S. et al. Observation of a solar flare at the limb with the YOHKOH Soft X-ray Telescope. Pub. Astron. Soc. Japan 44, L63-L69 (1992).

[12] Tsuneta, S. Structure and Dynamics of Magnetic Reconnection in a Solar Flare. Astrophys. J. 456, 840 (1996).

[13] Shibata, K. Evidence of Magnetic Reconnection in Solar Flares and a Unified Model of Flares. Astrophys. Space Sci. 264, 129-144 (1999).

[14] Lin, J. \& Forbes, T. G. Effects of reconnection on the coronal mass ejection process. J. Geophys. Res. 105, 2375-2392 (2000).

[15] Yokoyama, T., Akita, K., Morimoto, T., Inoue, K. \& Newmark, J. Clear Evidence of Reconnection Inflow of a Solar Flare. Astrophys. J. Lett. 546, L69-L72 (2001).

[16] Sui, L. \& Holman, G. D. Evidence for the Formation of a Large-Scale Current Sheet in a Solar Flare. Astrophys. J. Lett. 596, L251-L254 (2003).

[17] Lin, J. et al. Direct Observations of the Magnetic Reconnection Site of an Eruption on 2003 November 18. Astrophys. J. 622, 1251-1264 (2005). 
[18] Savage, S. L. et al. Low-altitude Reconnection Inflow-Outflow Observations during a 2010 November 3 Solar Eruption. Astrophys. J. 754, 13 (2012). 1111.1945.

[19] Su, Y. et al. Imaging coronal magnetic-field reconnection in a solar flare. Nature Physics 9, 489-493 (2013). 1307.4527.

[20] Yan, X. L. et al. Simultaneous Observation of a Flux Rope Eruption and Magnetic Reconnection during an X-class Solar Flare. Astrophys. J. Lett. 853, L18 (2018). 1801.02738.

[21] Takasao, S., Asai, A., Isobe, H. \& Shibata, K. Simultaneous Observation of Reconnection Inflow and Outflow Associated with the 2010 August 18 Solar Flare. Astrophys. J. Lett. 745, L6 (2012). 1112.1398.

[22] Gou, T., Liu, R., Kliem, B., Wang, Y. \& Veronig, A. M. The Birth of A Coronal Mass Ejection. Science Advances 5, 7004 (2019). 1811.04707.

[23] Cheng, X. et al. Observations of Turbulent Magnetic Reconnection within a Solar Current Sheet. Astrophys. J. 866, 64 (2018). 1808.06071.

[24] Xue, Z. et al. Observing the release of twist by magnetic reconnection in a solar filament eruption. Nature Communications 7, 11837 (2016).

[25] Sun, J. Q. et al. Extreme ultraviolet imaging of three-dimensional magnetic reconnection in a solar eruption. Nature Communications 6, 7598 (2015). 1506.08255.

[26] Masuda, S., Kosugi, T., Hara, H., Tsuneta, S. \& Ogawara, Y. A looptop hard X-ray source in a compact solar flare as evidence for magnetic reconnection. Nature 371, 495-497 (1994).

[27] Arnold, H. et al. Electron Acceleration during Macroscale Magnetic Reconnection. arXiv e-prints arXiv:2011.01147 (2020). 2011.01147.

[28] Warmuth, A. \& Mann, G. Thermal-nonthermal energy partition in solar flares derived from X-ray, EUV, and bolometric observations. Discussion of recent studies. Astron. Astrophys. 644, A172 (2020). 2011.04442. 
[29] Innes, D. E., Guo, L.-J., Huang, Y.-M. \& Bhattacharjee, A. IRIS Si IV Line Profiles: An Indication for the Plasmoid Instability during Smallscale Magnetic Reconnection on the Sun. Astrophys. J. 813, 86 (2015). 1509.08837.

[30] Guo, L.-J., De Pontieu, B., Huang, Y.-M., Peter, H. \& Bhattacharjee, A. Observations and Modeling of the Onset of Fast Reconnection in the Solar Transition Region. Astrophys. J. 901, 148 (2020). 2009.11475.

[31] Kliem, B., Karlický, M. \& Benz, A. O. Solar flare radio pulsations as a signature of dynamic magnetic reconnection. Astron. Astrophys. 360, 715-728 (2000). astro-ph/0006324.

[32] French, R. J., Judge, P. G., Matthews, S. A. \& van Driel-Gesztelyi, L. Spectropolarimetric Insight into Plasma Sheet Dynamics of a Solar Flare. Astrophys. J. Lett. 887, L34 (2019). 1911.12666.

[33] Chitta, L. P. \& Lazarian, A. Onset of Turbulent Fast Magnetic Reconnection Observed in the Solar Atmosphere. Astrophys. J. Lett. 890, L2 (2020). 2001.08595.

[34] Tian, H. et al. Imaging and Spectroscopic Observations of Magnetic Reconnection and Chromospheric Evaporation in a Solar Flare. Astrophys. J. Lett. 797, L14 (2014). 1411.2301.

[35] Yang, S., Zhang, J. \& Xiang, Y. Magnetic Reconnection between Smallscale Loops Observed with the New Vacuum Solar Telescope. Astrophys. J. Lett. 798, L11 (2015). 1412.1314.

[36] Wang, T., Sui, L. \& Qiu, J. Direct Observation of High-Speed Plasma Outflows Produced by Magnetic Reconnection in Solar Impulsive Events. Astrophys. J. Lett. 661, L207-L210 (2007). 0709.2329.

[37] Yu, S. et al. Magnetic Reconnection during the Post-impulsive Phase of a Long-duration Solar Flare: Bidirectional Outflows as a Cause of Microwave and X-Ray Bursts. Astrophys. J. 900, 17 (2020). 2007.10443.

[38] Burch, J. L. et al. Electron-scale measurements of magnetic reconnection in space. Science 352, aaf2939 (2016). 
[39] Phan, T. D. et al. Electron magnetic reconnection without ion coupling in Earth's turbulent magnetosheath. Nature 557, 202-206 (2018).

[40] Liu, R., Chen, J., Wang, Y. \& Liu, K. Investigating Energetic X-Shaped Flares on the Outskirts of A Solar Active Region. Scientific Reports 6 , 34021 (2016). 1609.02713.

[41] Kawabata, Y., Inoue, S. \& Shimizu, T. Non-potential Field Formation in the X-shaped Quadrupole Magnetic Field Configuration. Astrophys. J. 842, 106 (2017). 1705.02560.

[42] Liu, Z. et al. New vacuum solar telescope and observations with high resolution. Res. Astron. Astrophys. 14, 705-718 (2014).

[43] Schou, J. et al. Design and Ground Calibration of the Helioseismic and Magnetic Imager (HMI) Instrument on the Solar Dynamics Observatory (SDO). Solar Phys. 275, 229-259 (2012).

[44] Xue, Z., Yan, X., Yang, L., Wang, J. \& Zhao, L. Observing Formation of Flux Rope by Tether-cutting Reconnection in the Sun. Astrophys. J. Lett. 840, L23 (2017).

[45] Lin, R. P. et al. The Reuven Ramaty High-Energy Solar Spectroscopic Imager (RHESSI). Solar Phys. 210, 3-32 (2002).

[46] Culhane, J. L. et al. The EUV Imaging Spectrometer for Hinode. Solar Phys. 243, 19-61 (2007).

[47] Bhattacharjee, A., Huang, Y.-M., Yang, H. \& Rogers, B. Fast reconnection in high-Lundquist-number plasmas due to the plasmoid Instability. Physics of Plasmas 16, 112102 (2009). 0906.5599.

[48] Ni, L., Kliem, B., Lin, J. \& Wu, N. Fast Magnetic Reconnection in the Solar Chromosphere Mediated by the Plasmoid Instability. Astrophys. J. 799, 79 (2015). 1509.06895.

[49] Dere, K. P., Landi, E., Mason, H. E., Monsignori Fossi, B. C. \& Young, P. R. CHIANTI - an atomic database for emission lines. Astron. Astrophys. Suppl. 125, 149-173 (1997). 
[50] Landi, E. et al. CHIANTI-An Atomic Database for Emission Lines. VII. New Data for X-Rays and Other Improvements. Astrophys. J. Suplement Series 162, 261-280 (2006).

[51] Labrosse, N. et al. Physics of Solar Prominences: I-Spectral Diagnostics and Non-LTE Modelling. Space Sci. Rev. 151, 243-332 (2010). 1001.1620 .

[52] Daughton, W. et al. Role of electron physics in the development of turbulent magnetic reconnection in collisionless plasmas. Nature Physics 7, 539-542 (2011).

[53] Longcope, D., Unverferth, J., Klein, C., McCarthy, M. \& Priest, E. Evidence for Downflows in the Narrow Plasma Sheet of 2017 September 10 and Their Significance for Flare Reconnection. Astrophys. J. 868, 148 (2018).

[54] Lemen, J. R. et al. The Atmospheric Imaging Assembly (AIA) on the Solar Dynamics Observatory (SDO). Solar Phys. 275, 17-40 (2012).

[55] Krucker, S. \& Battaglia, M. Particle Densities within the Acceleration Region of a Solar Flare. Astrophys. J. 780, 107 (2014).

[56] Kliem, B. Particle orbits, trapping, and acceleration in a filamentary current sheet model. Astrophys. J. Suplement Series 90, 719-728 (1994).

[57] Drake, J. F., Swisdak, M., Che, H. \& Shay, M. A. Electron acceleration from contracting magnetic islands during reconnection. Nature 443, 553-556 (2006).

[58] Titov, V. S., Hornig, G. \& Démoulin, P. Theory of magnetic connectivity in the solar corona. Journal of Geophysical Research (Space Physics) 107, 1164 (2002).

[59] Priest, E. R. \& Démoulin, P. Three-dimensional magnetic reconnection without null points. 1. Basic theory of magnetic flipping. J. Geophys. Res. 100, 23443-23464 (1995).

[60] Ji, H. \& Daughton, W. Phase diagram for magnetic reconnection in heliophysical, astrophysical, and laboratory plasmas. Physics of Plasmas 18, 111207-111207 (2011). 1109.0756. 
[61] Schuck, P. W. Tracking Magnetic Footpoints with the Magnetic Induction Equation. Astrophys. J. 646, 1358-1391 (2006).

[62] Li, T. \& Zhang, J. Slipping Magnetic Reconnection Triggering a Solar Eruption of a Triangle-shaped Flag Flux Rope. Astrophys. J. Lett. 791, L13 (2014). 1407.4180.

[63] Xiang, Y.-y., Liu, Z. \& Jin, Z.-y. High resolution reconstruction of solar prominence images observed by the New Vacuum Solar Telescope. New Astron 49, 8-12 (2016).

[64] Bobra, M. G. et al. The Helioseismic and Magnetic Imager (HMI) Vector Magnetic Field Pipeline: SHARPs - Space-Weather HMI Active Region Patches. Solar Phys. 289, 3549-3578 (2014). 1404.1879.

[65] Centeno, R. et al. The Helioseismic and Magnetic Imager (HMI) Vector Magnetic Field Pipeline: Optimization of the Spectral Line Inversion Code. Solar Phys. 289, 3531-3547 (2014). 1403.3677.

[66] Borrero, J. M. et al. VFISV: Very Fast Inversion of the Stokes Vector for the Helioseismic and Magnetic Imager. Solar Phys. 273, 267-293 (2011). 0901.2702.

[67] Metcalf, T. R. Resolving the 180-degree ambiguity in vector magnetic field measurements: The 'minimum' energy solution. Solar Phys. 155, 235-242 (1994).

[68] Leka, K. D. et al. Resolving the $180^{\mathrm{deg}}$ Ambiguity in Solar Vector Magnetic Field Data: Evaluating the Effects of Noise, Spatial Resolution, and Method Assumptions. Solar Phys. 260, 83-108 (2009).

[69] Sun, X. On the Coordinate System of Space-Weather HMI Active Region Patches (SHARPs): A Technical Note. ArXiv e-prints (2013). 1309.2392.

[70] Jiang, C., Wu, S. T., Feng, X. \& Hu, Q. Data-driven magnetohydrodynamic modelling of a flux-emerging active region leading to solar eruption. Nature Communications 7, 11522 (2016).

[71] Jiang, C. et al. Reconstruction of a Large-scale Pre-flare Coronal Current Sheet Associated with a Homologous X-shaped Flare. Astrophys. J. 850, 8 (2017). 1710.02775. 
[72] Wiegelmann, T. Optimization code with weighting function for the reconstruction of coronal magnetic fields. Solar Phys. 219, 87-108 (2004). 0802.0124.

[73] Wiegelmann, T., Inhester, B. \& Sakurai, T. Preprocessing of Vector Magnetograph Data for a Nonlinear Force-Free Magnetic Field Reconstruction. Solar Phys. 233, 215-232 (2006). astro-ph/0612641.

[74] Schwartz, R. A. et al. RHESSI Data Analysis Software: Rationale and Methods. Solar Phys. 210, 165-191 (2002).

[75] Liu, Y., Zhao, J. \& Schuck, P. W. Horizontal Flows in the Photosphere and Subphotosphere of Two Active Regions. Solar Phys. 287, 279-291 (2013).

\section{Acknowledgements}

We would like to thank the NVST, SDO/AIA, SDO/HMI, GOES, RHESSI, and Hinode teams for high-cadence data support. This work is sponsored by the National Science Foundation of China (NSFC) under the grant numbers (11873087, 11973084, 11803085, 2019FD085, 12003064, 11633008, 11763004, U1831210, 11803002), by the Yunnan Key Science Foundation of China under numbers (2018FA001, 2018FB007) and Yunnan Science Foundation for Distinguished Young Scholars, by Project Supported by the Specialized Research Fund for State Key Laboratories, by Key Research and Development Project of Yunnan Province202003AD150019, and by the joint NSFC/DFG Grant 41761134088/KL817.8-1.

\section{Author contributions}

X.L.Y. developed the ideas, performed analysis of the data, and lead the discussion of the manuscript. Z.K.X., L.H.Y., J.C.W., D.F.K., and Y.L.S. performed analysis of the data. C.W.J. carried out the MHD simulation. E.R.P. evaluated the energy of magnetic reconnection. B.K. contributed to the interpretation of the data and to the discussion. X.S.F. and Z.L. contributed to the discussion. All authors reviewed the manuscript.

\section{Additional information}

Correspondence and requests for materials should be addressed to X.L.Y. and C.W.J (email: yanxl@ynao.ac.cn; chaowei@hit.edu.cn).

Competing financial interests: The authors declare no competing financial interests. 

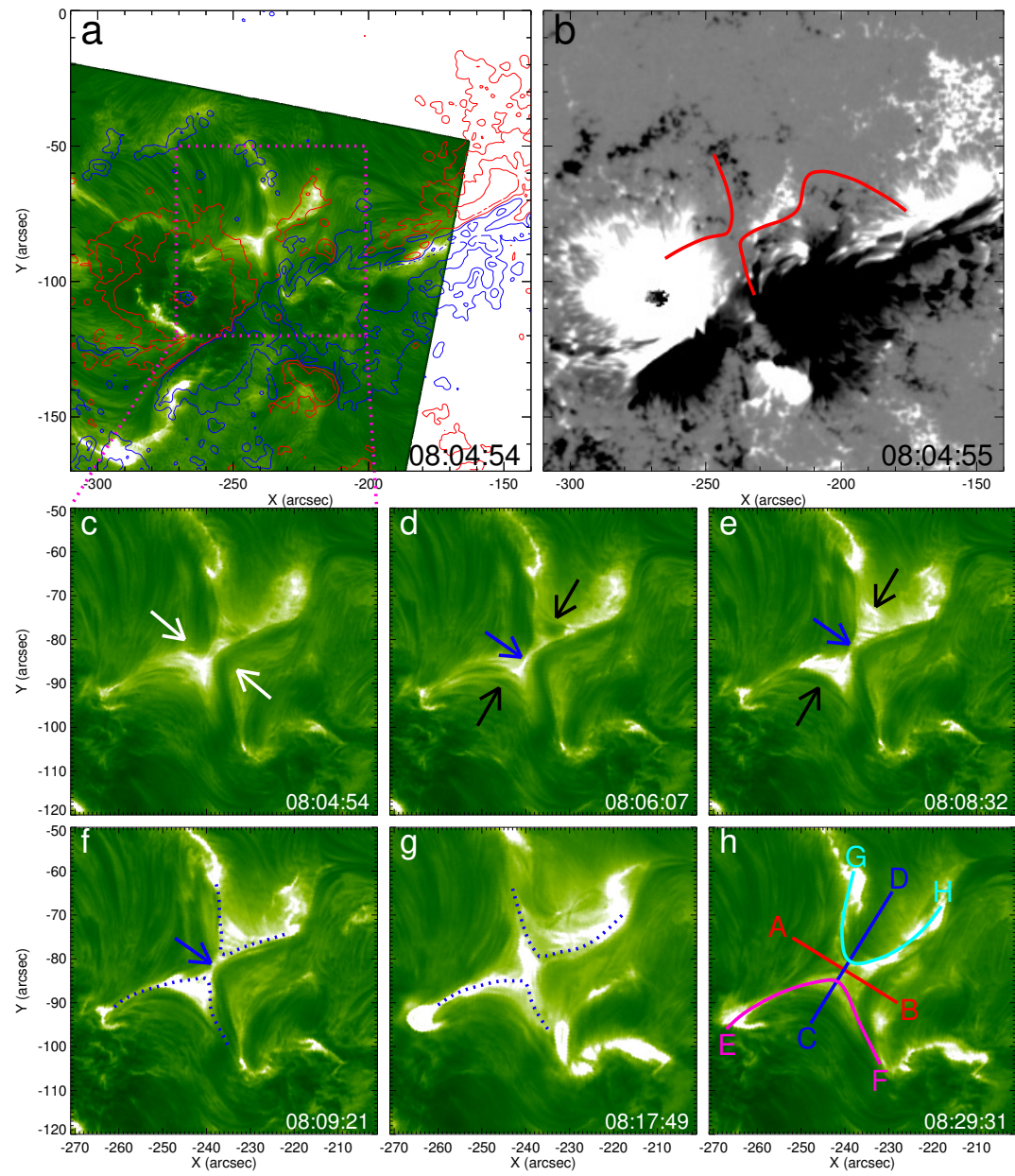

Figure 1: Magnetic reconnection during the confined flare, observed in the center of the $\mathbf{H} \alpha$ line. (a) $H \alpha$ image observed at 08:04:54 UT by NVST superimposed on the line-of-sight magnetogram observed by SDO/HMI. Red (blue) contours indicate positive (negative) polarity of $\pm 200 \mathrm{G}$ and $\pm 800 \mathrm{G}$. (b) Line-of-sight magnetogram observed by SDO/HMI at 08:04:55 UT. The two curved red lines indicate the chromospheric fibrils and the active-region filament participating in the reconnection. ( $\mathrm{c}-\mathrm{h})$ The process of reconnection as seen in the $H \alpha$ observations. White arrows in $\mathbf{c}$ indicate the two reconnecting structures. Black arrows in $\mathbf{d}$ and $\mathbf{e}$ indicate the new chromospheric fibrils formed by reconnection. Blue arrows in $\mathbf{d}-\mathbf{f}$ indicate the current sheet. The dotted lines in $\mathbf{f}$ and $\mathbf{g}$ indicate the cusp-shaped structures (magnetic separatrices) formed between the reconnection in- and outflows. 

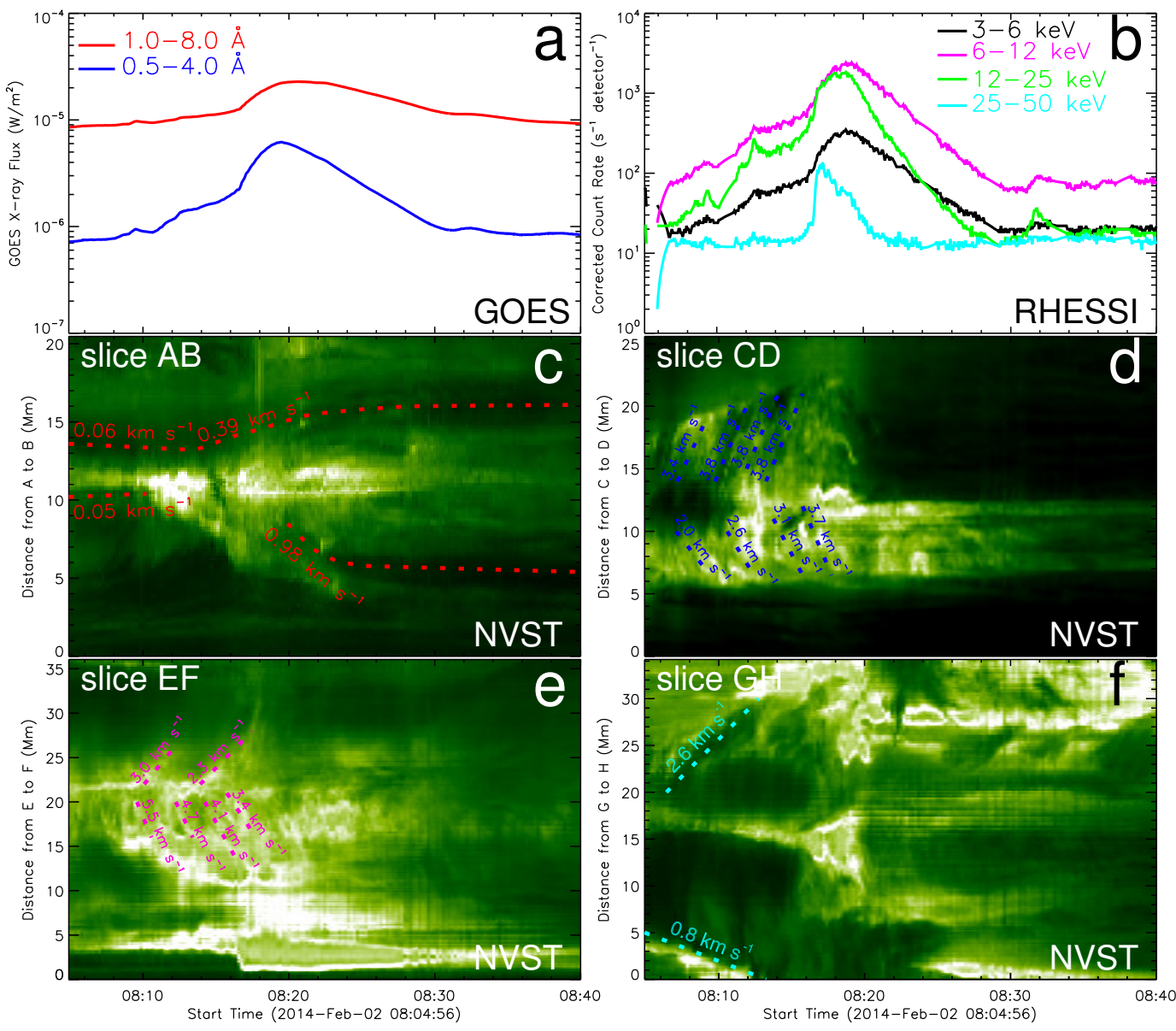

Figure 2: Profiles of GOES X-ray flux, inflows, and outflows during reconnection. (a) The profile of GOES X-rays in 1-8 $\AA$ (red line) and $0.5-$ $4 \AA$ (blue line). (b) The profile of the RHESSI X-ray counts in the 3-6, 6-12, 12-25, and 25-50 keV bands. (c) Inflow (dotted curve) along the A-B slit inferred from time-slices of the intensity. (d) Outflow along the C-D slit. (e) Outflow along the curved line E-F. (f) Outflow along the curved line G-H. All these lines are marked in Fig. 1h. 


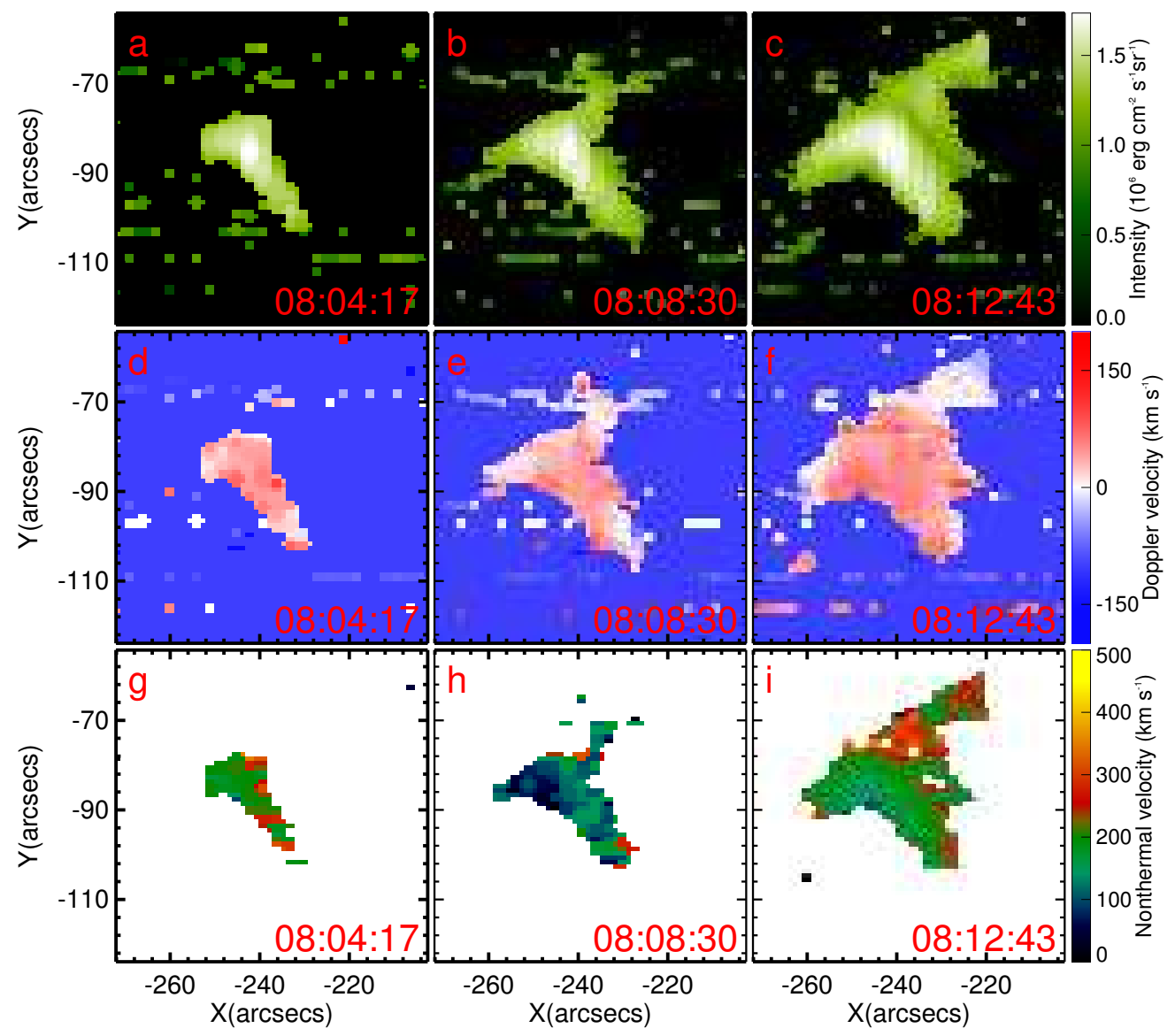

Figure 3: Hinode/EIS observation at Fe XVII 255 Å during magnetic reconnection. $(\mathrm{a}-\mathrm{c})$ Evolution of the cusp-shaped structure in the intensity images. (d-f) Doppler velocity and ( $\mathrm{g}-\mathrm{i})$ non-thermal velocity obtained from single Gaussian fitting. 

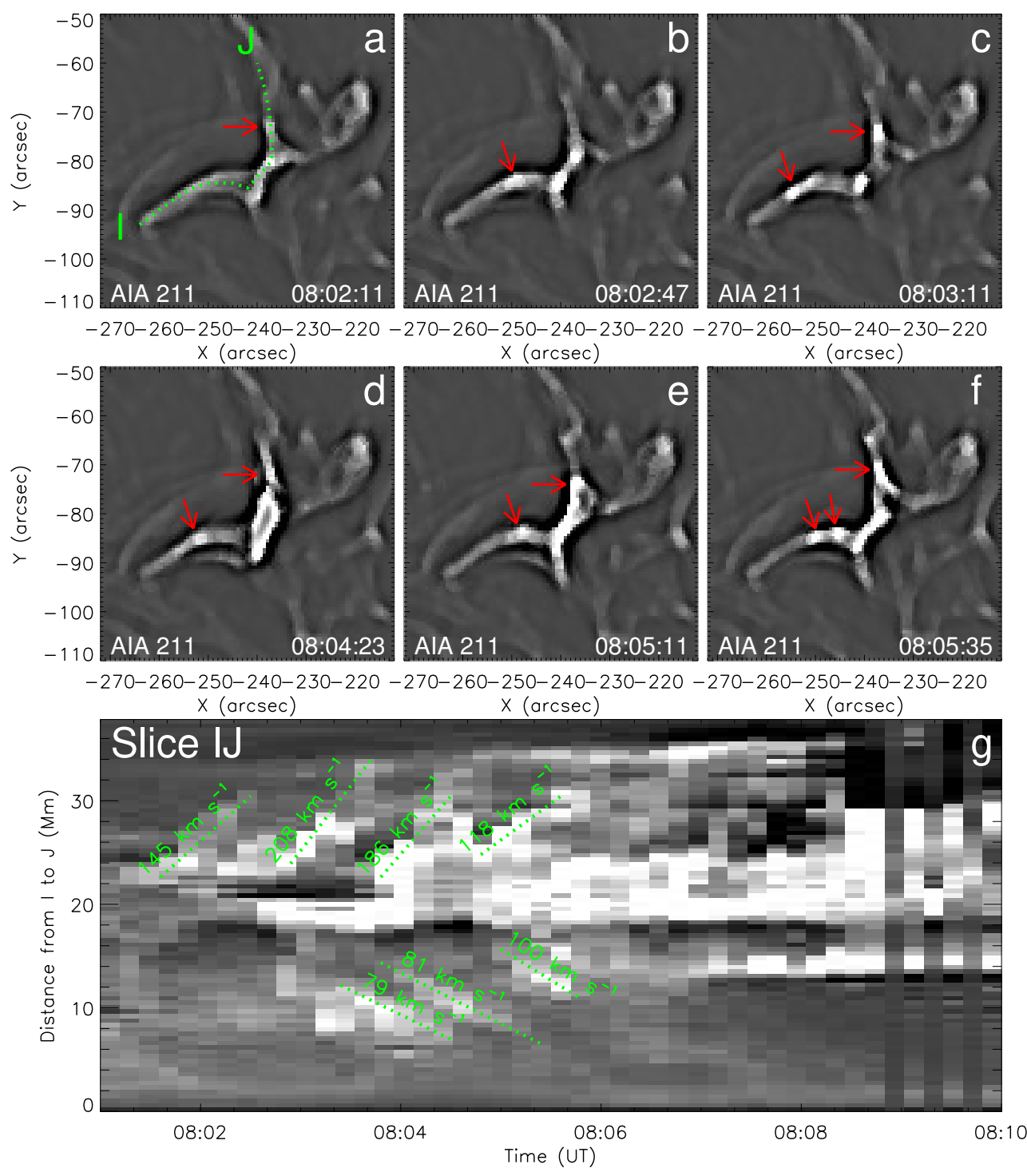

Figure 4: Fine structures in the SDO/AIA $211 \AA \AA$ images revealed by the unsharp masking technique. Red arrows mark the different bright blobs moving from the reconnection region (Fig. 4a-f). The green-dotted line in Fig. 4a indicates the trajectory I-J used to make the time-distance diagram of Fig. 4g. The projected velocities of the blobs lie in the range from $79 \mathrm{~km} \mathrm{~s}^{-1}$ to $208 \mathrm{~km} \mathrm{~s}^{-1}$. 


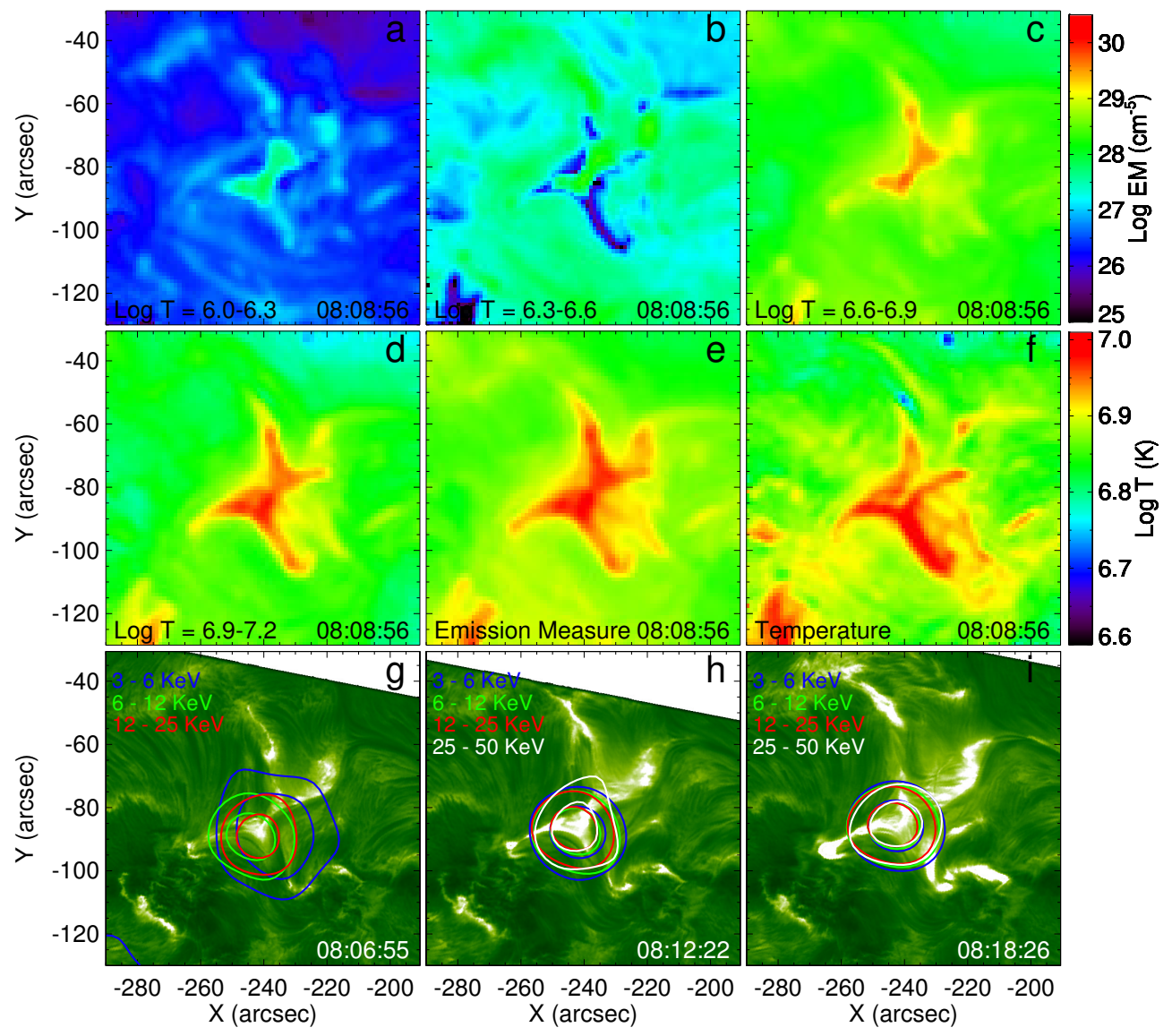

Figure 5: Emission measure and temperature maps and $\mathrm{H} \alpha$ images with RHESSI X-ray sources superimposed. (a-d): DEM maps in four temperature ranges. (e) Total emission measure. (f) Temperature of the reconnection region derived from six AIA wavelengths. (g-i): $\mathrm{H} \alpha$ images with RHESSI hard X-ray sources superimposed. The levels of the contours are at $50 \%$ and $80 \%$ of the peak values in the $3-6,6-12,12-25$, and $25-50 \mathrm{keV}$ bands. 

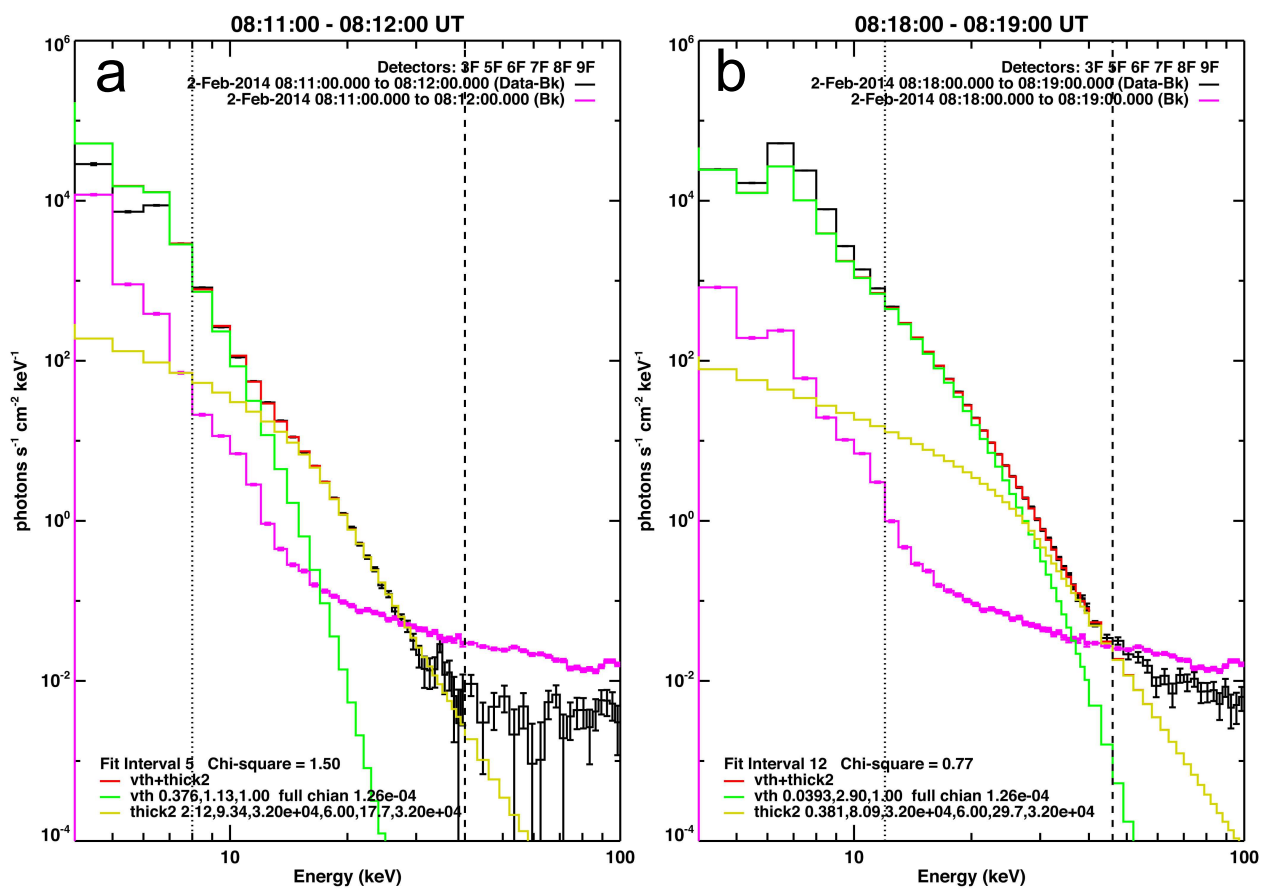

Figure 6: RHESSI photon spectra accumulated around 08:12 UT and 08:19 UT during the quoted 1-minute intervals on 2014 February 2 . The spectra are fitted by combining the vth and thick2 functions (see Methods). The black line shows the photon spectrum with the background subtracted. The pink line shows the background. The green and the yellow lines display the fitting with the single-temperature thermal model and the thick-target nonthermal model, respectively. 

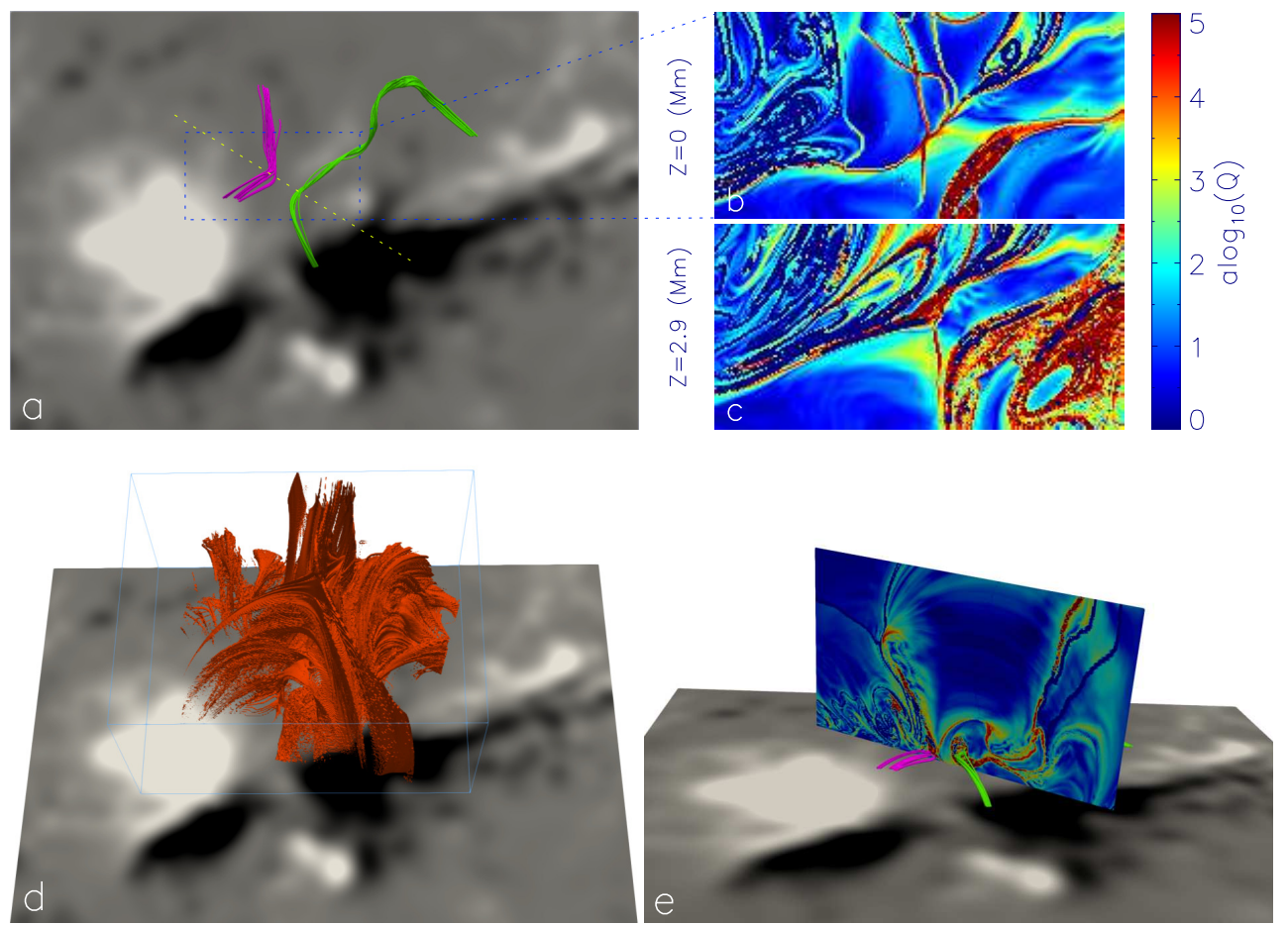

Figure 7: Magnetic structure of the source region. (a) Selected field lines showing the twisted magnetic structure of the filament (green) and the loop-like structure of the chromospheric fibrils (red) before the reconnection event, obtained by using NLFFF extrapolation from the vector magnetogram. (b)-(c) Map of the magnetic squashing degree $\log Q$ at heights $Z=0$ and $Z=2.9 \mathrm{Mm}$ above the photosphere. (d) Iso-surface $\log Q=4$ in the reconnection region. (e) $\log Q$ in the vertical cross-section marked by a yellow dashed line in (a). 

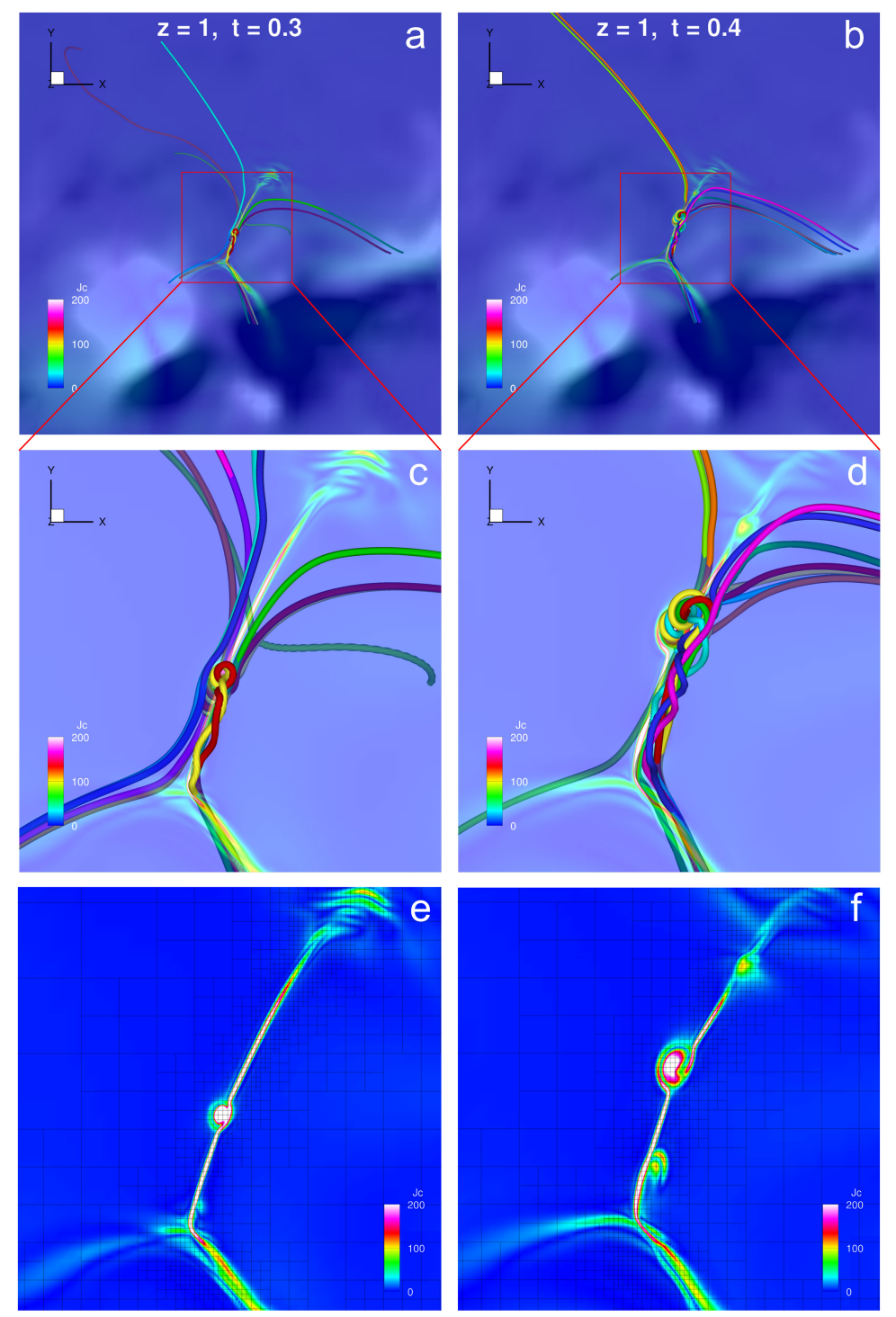

Figure 8: Evolution of magnetic field, current sheet, and plasmoids (blobs) during magnetic reconnection in the data-driven MHD simulation. (a) and (b): Evolution of the magnetic structure involved in the modeled reconnection event. (c) and (d): Zoom showing the magnetic structure of the current sheet at two selected times. The red boxes in (a) and (b) show the field of view of (c)-(f). (e) and (f): Distribution of electric current density $(\boldsymbol{J} \boldsymbol{c})$ in the current sheet (also overlaid in (c)-(d)) at the height $z=1$ (11.6 Mm). 

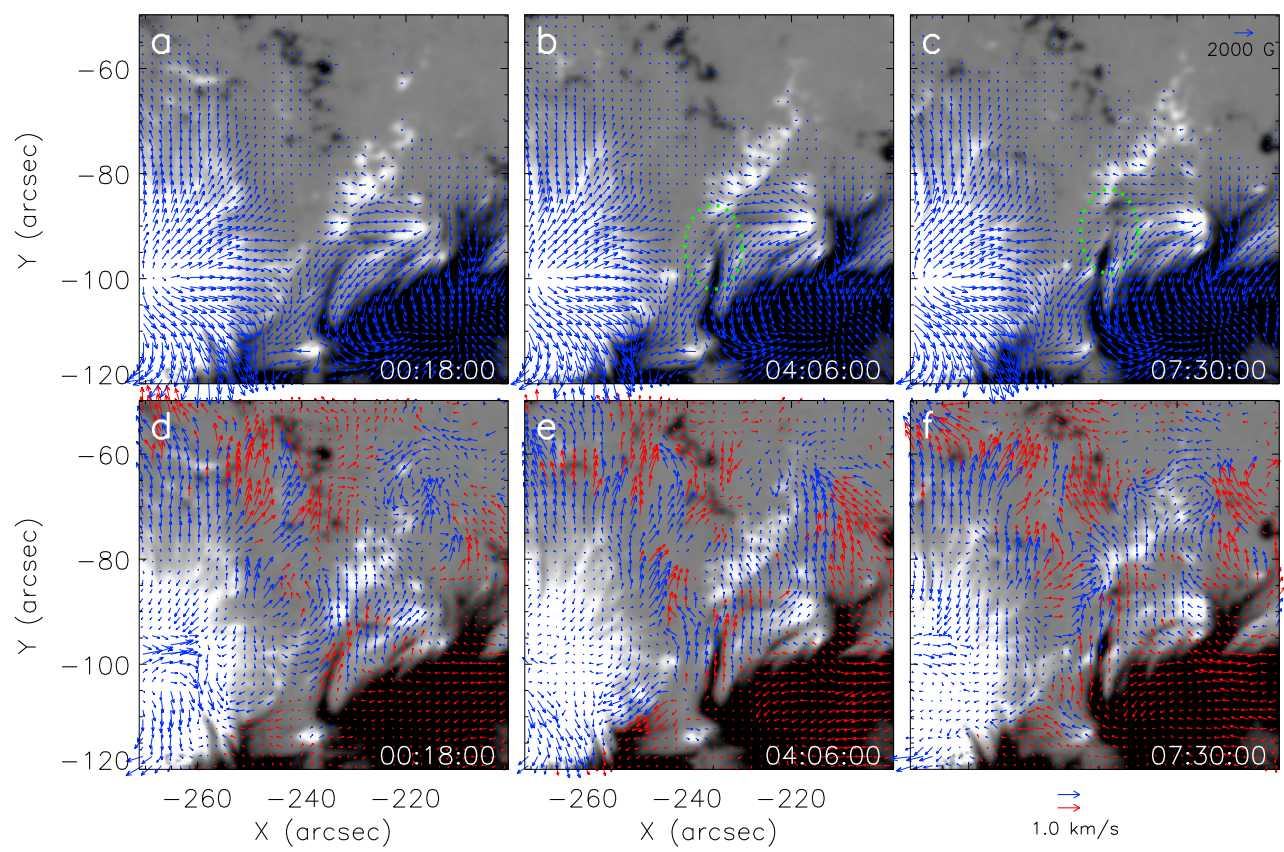

Figure 9: Evolution of the photospheric vector magnetic field observed by SDO/HMI and flow field derived using the DAVE algorithm. $(\mathrm{a}-\mathrm{c})$ : Vector magnetograms. The greyscale images show the vertical field component, saturated at $1000 \mathrm{G}$, and the arrows show the horizontal component. (d-f): Evolution of the flow field. Blue and red arrows indicate the horizontal photospheric velocity in the positive and negative polarities, respectively. 

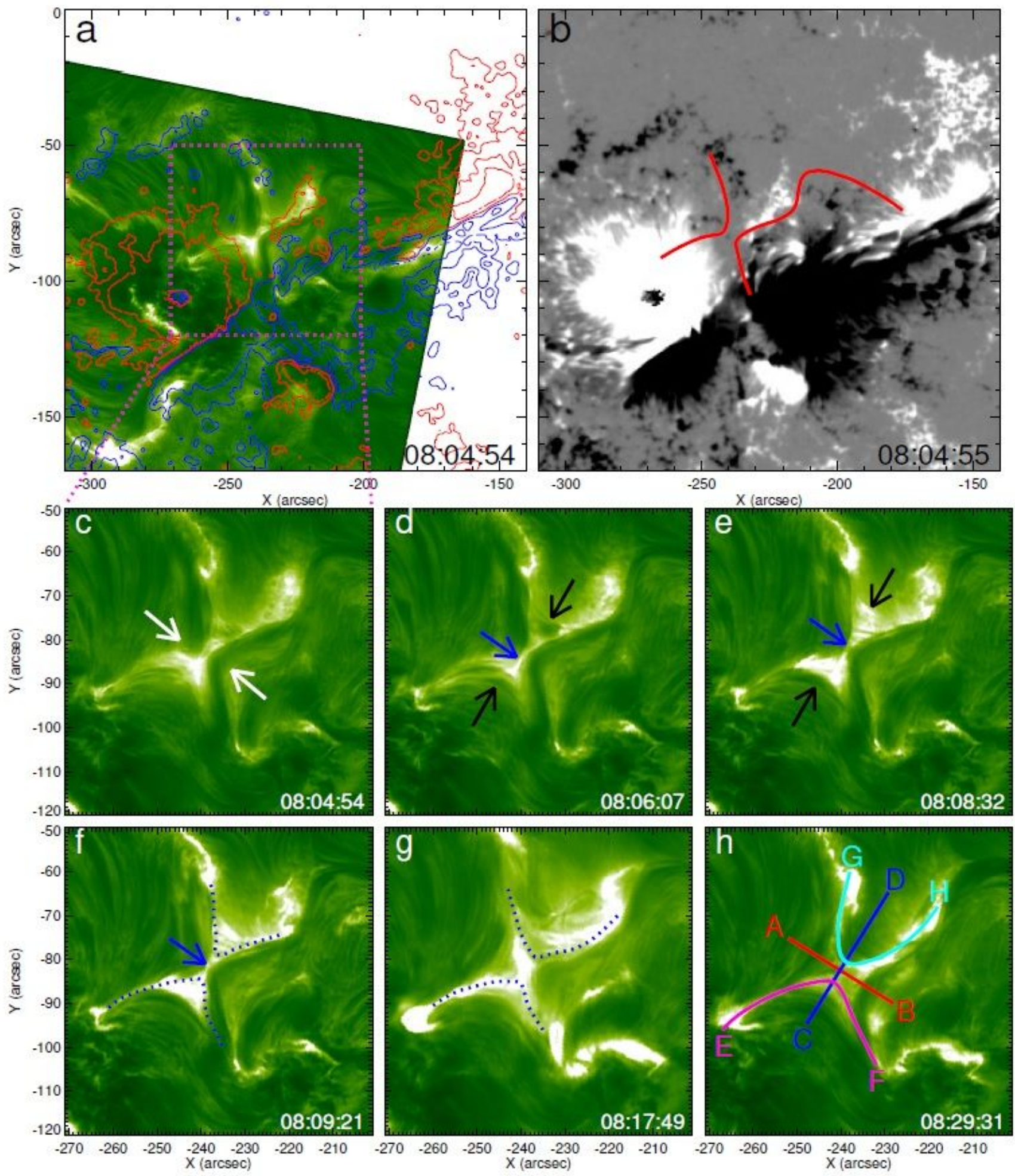

\section{Figure 1}

Magnetic reconnection during the confined are, observed in the center of the Ha line. (see full caption in Manuscript file) 

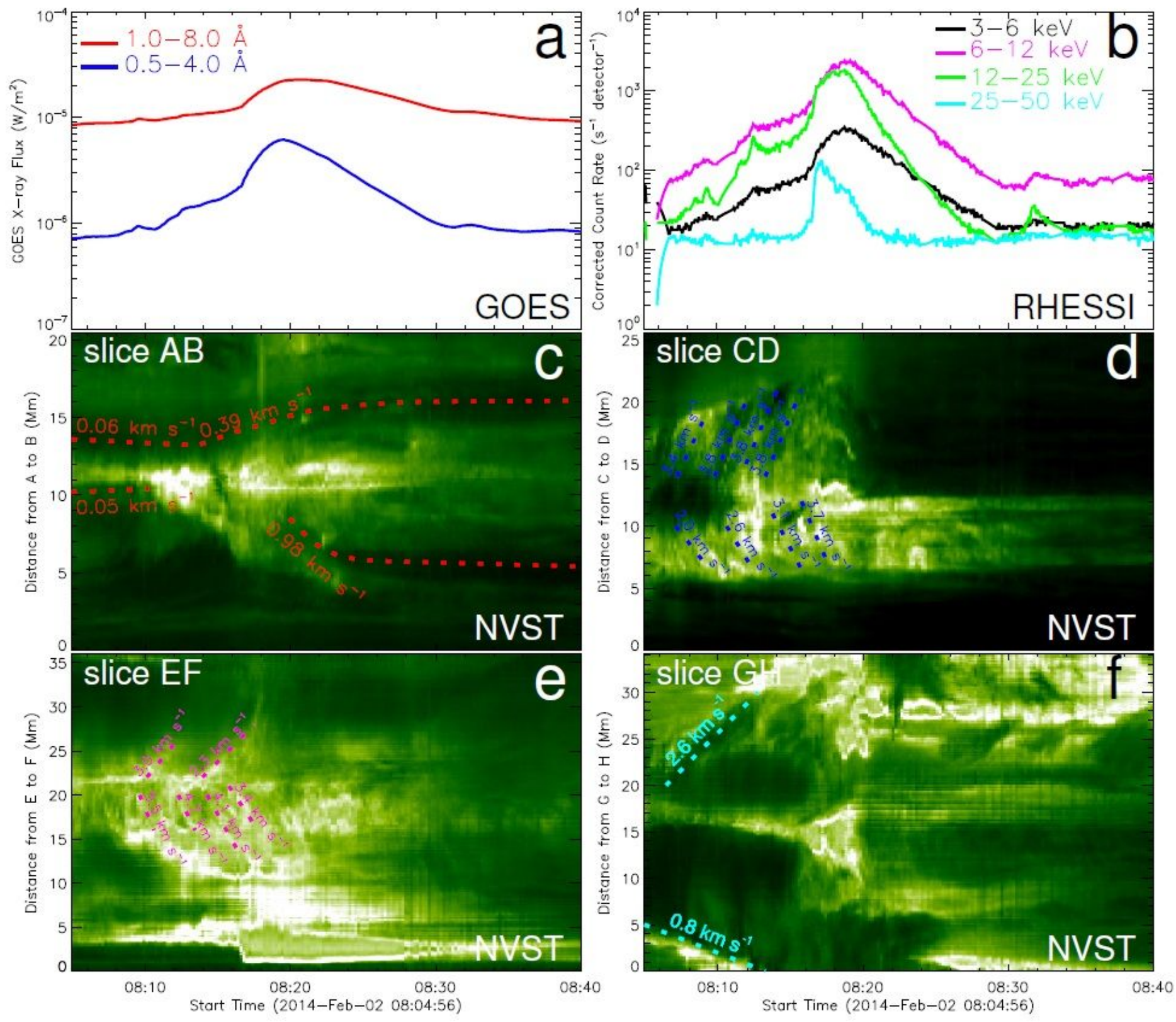

Figure 2

Proles of GOES X-ray flux, in flows, and out flows during reconnection. (see full caption in Manuscript file) 


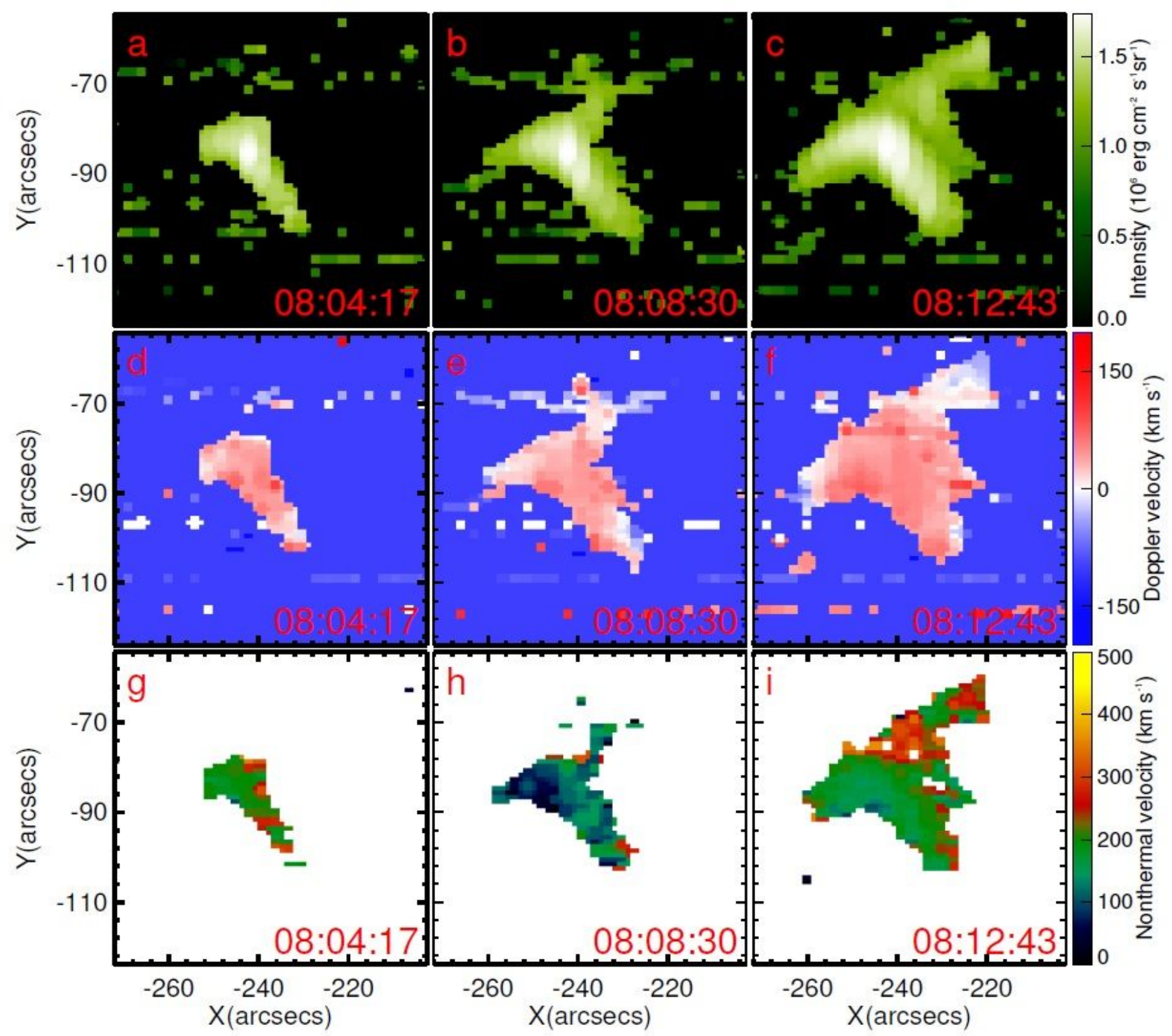

Figure 3

Hinode/EIS observation at Fe XVII 255A during magnetic reconnection. (see full caption in Manuscript file) 

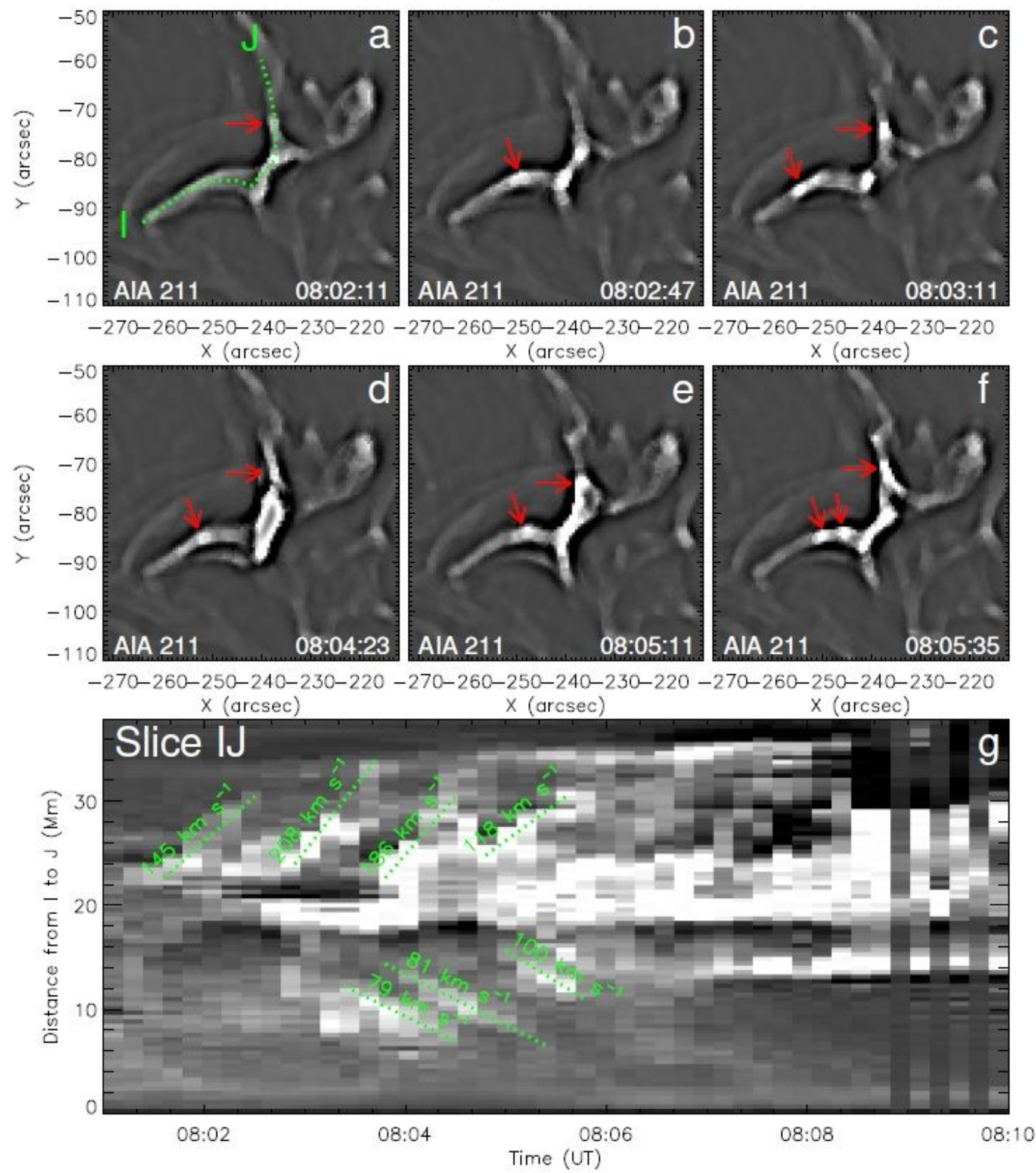

\section{Figure 4}

Fine structures in the SDO/AIA $211 \mathrm{~A}$ images revealed by the unsharp masking technique. (see full caption in Manuscript file) 


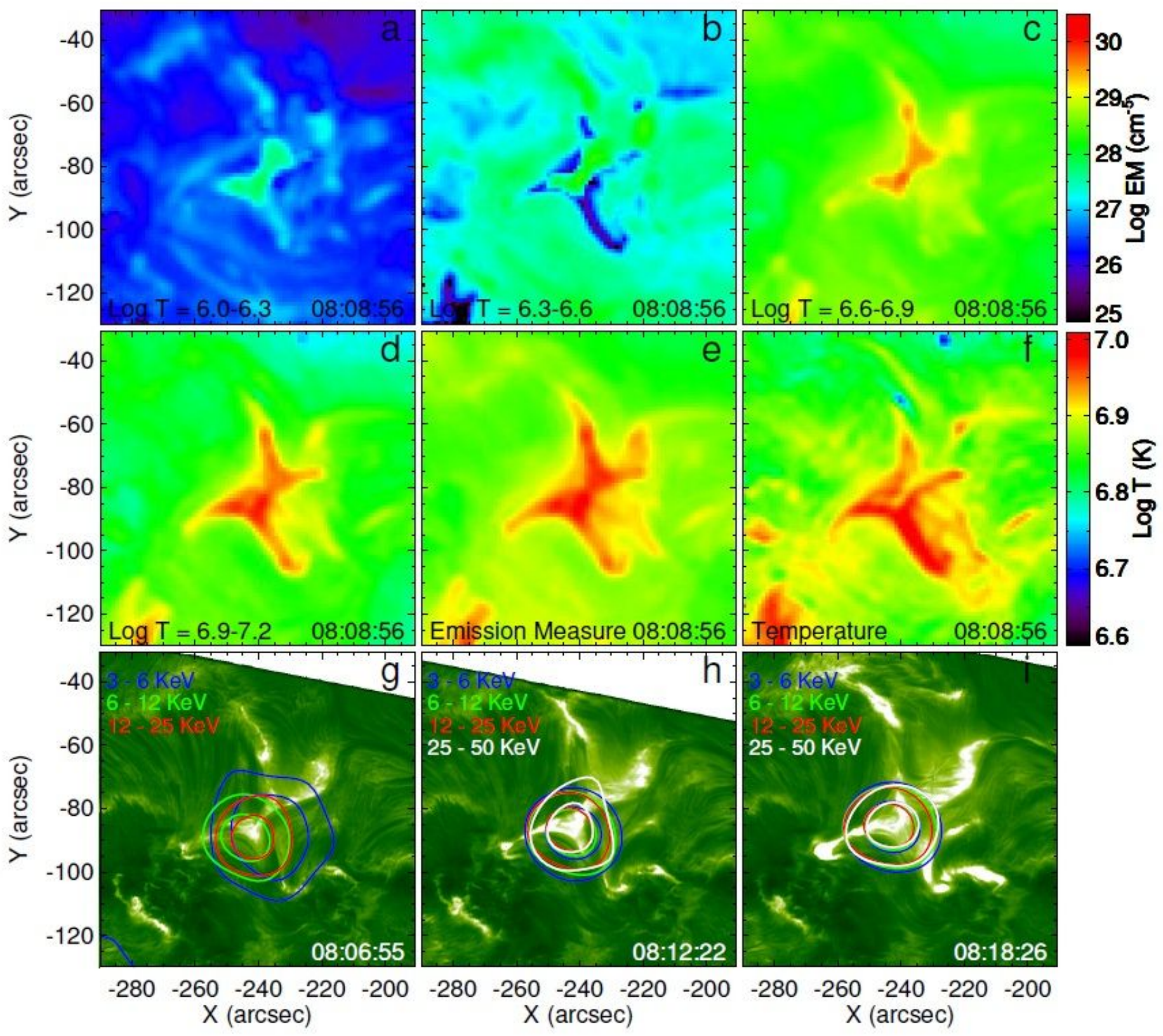

Figure 5

Emission measure and temperature maps and Ha images with RHESSI X-ray sources superimposed. (see full caption in Manuscript file) 

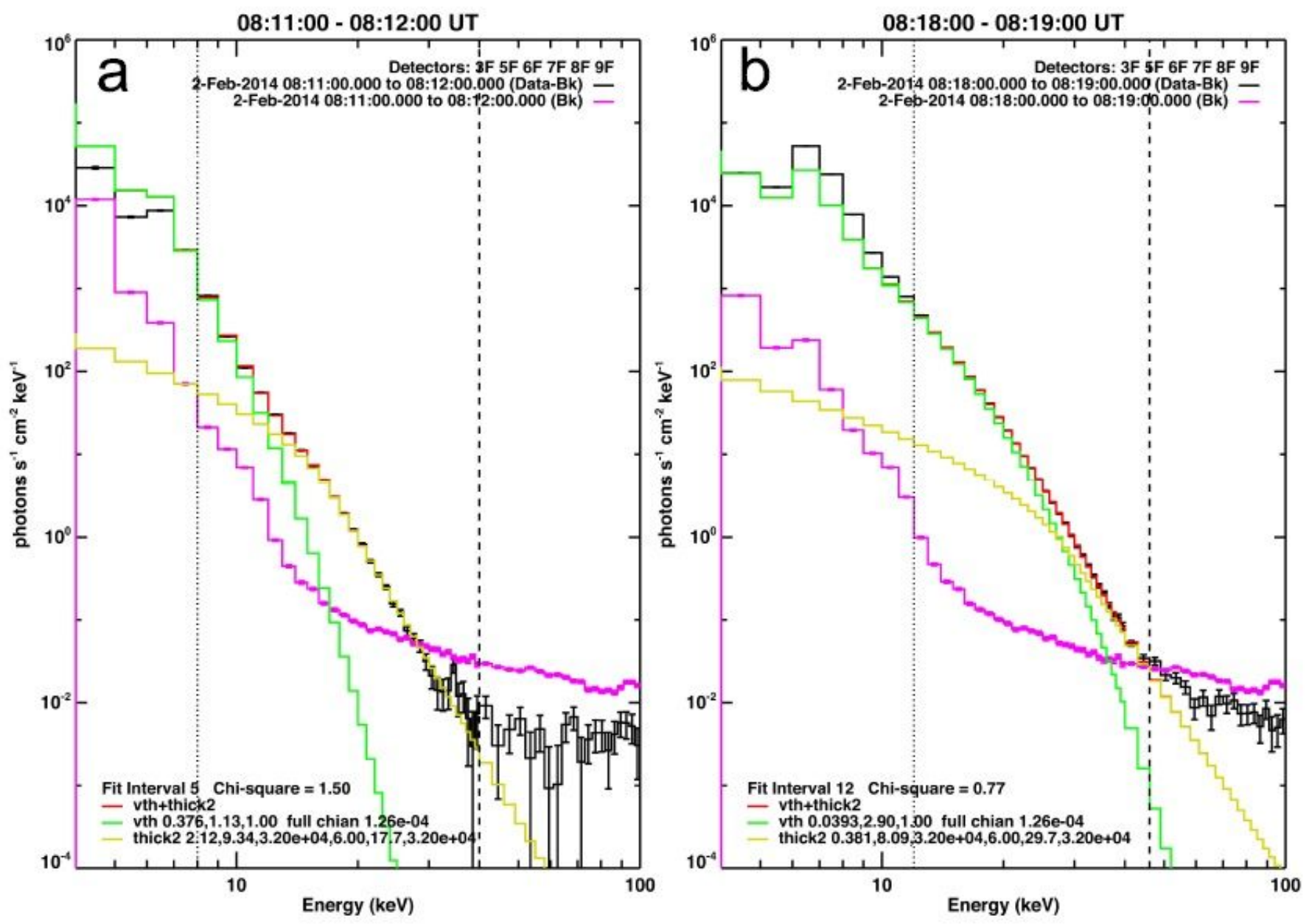

Figure 6

RHESSI photon spectra accumulated around 08:12 UT and 08:19 UT during the quoted 1-minute intervals on 2014 February 2. (see full caption in Manuscript file) 

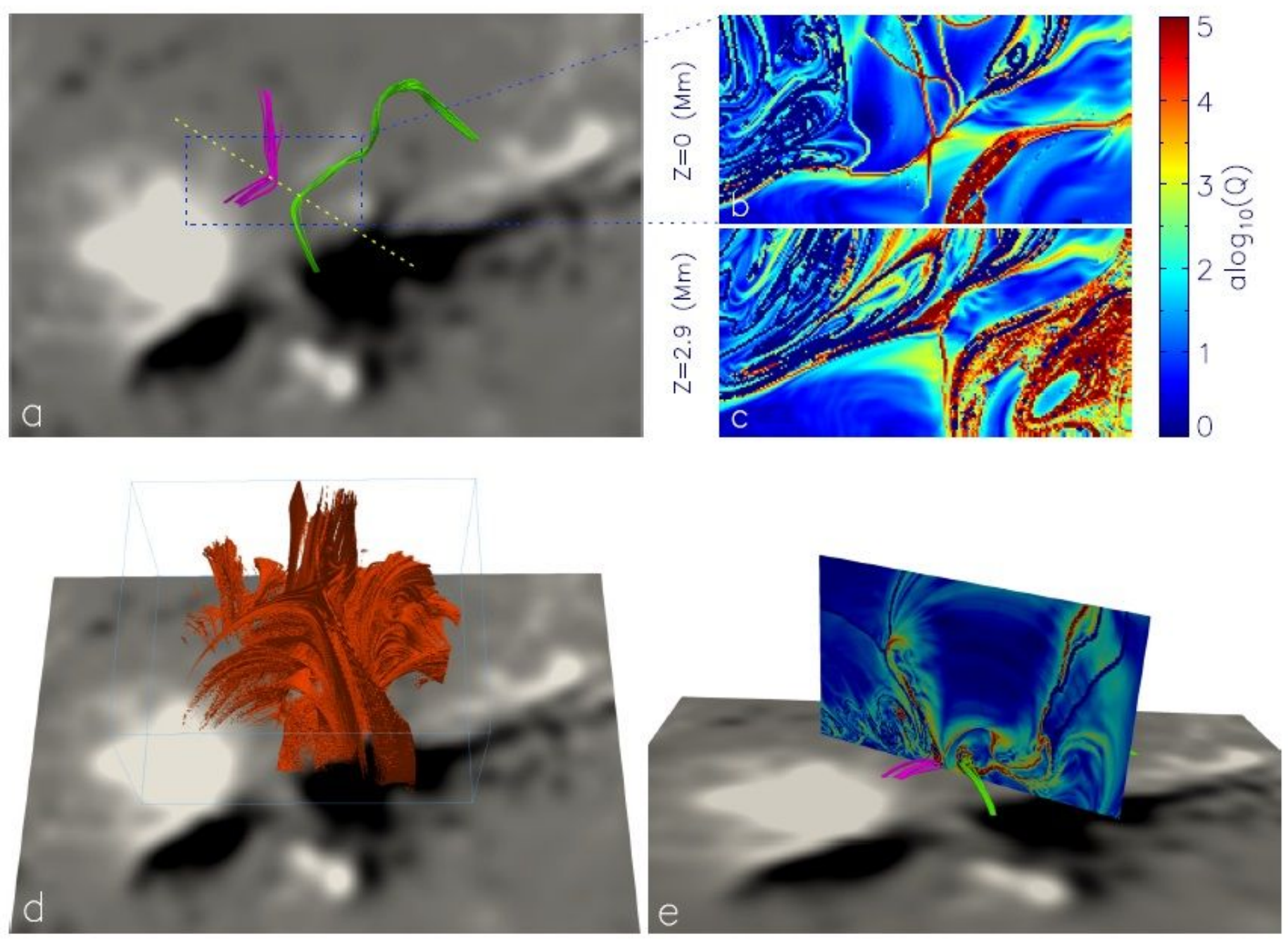

Figure 7

Magnetic structure of the source region. (see full caption in Manuscript file) 

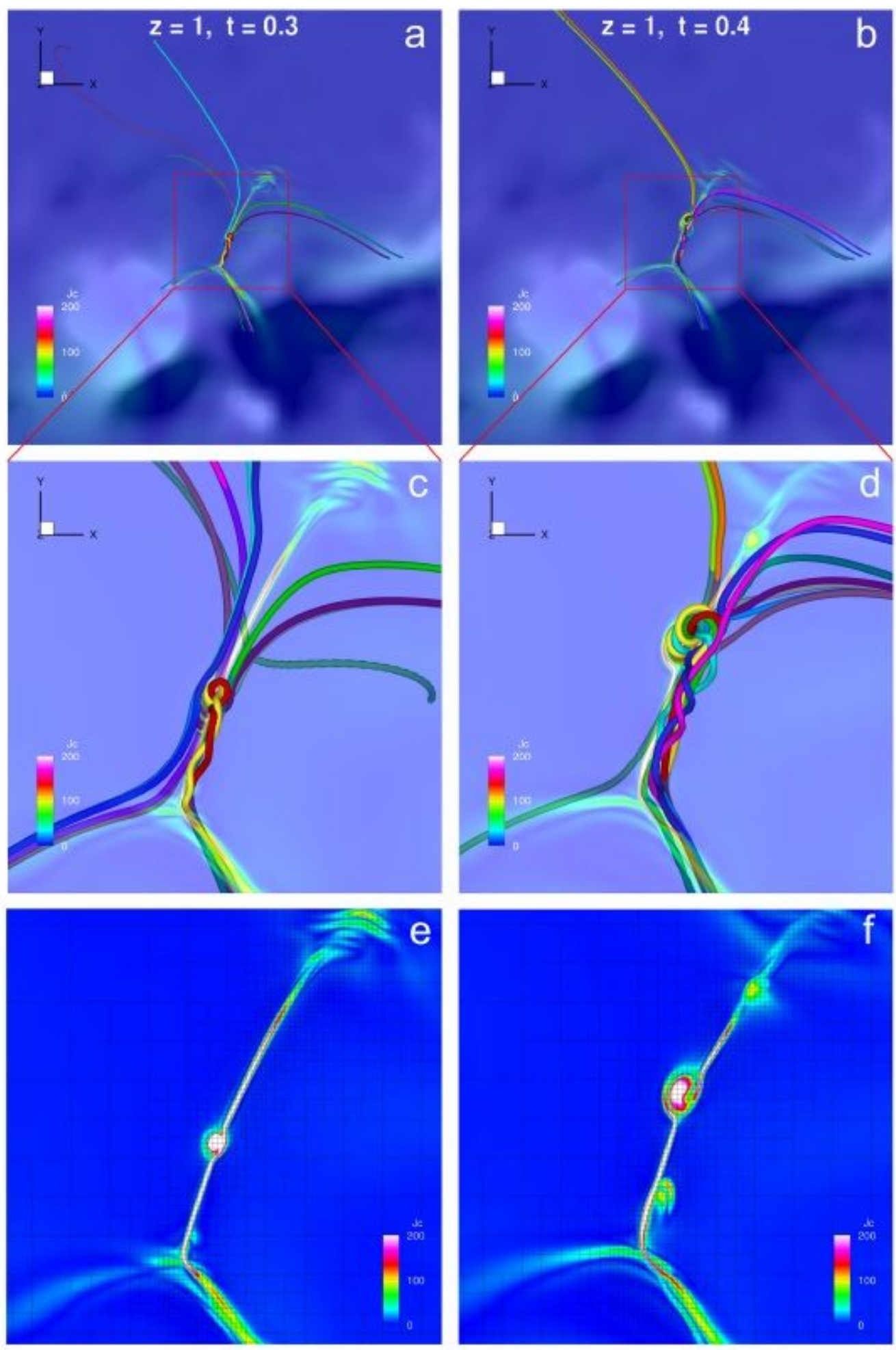

\section{Figure 8}

Evolution of magnetic field, current sheet, and plasmoids (blobs) during magnetic reconnection in the data-driven MHD simulation. (see full caption in Manuscript file) 


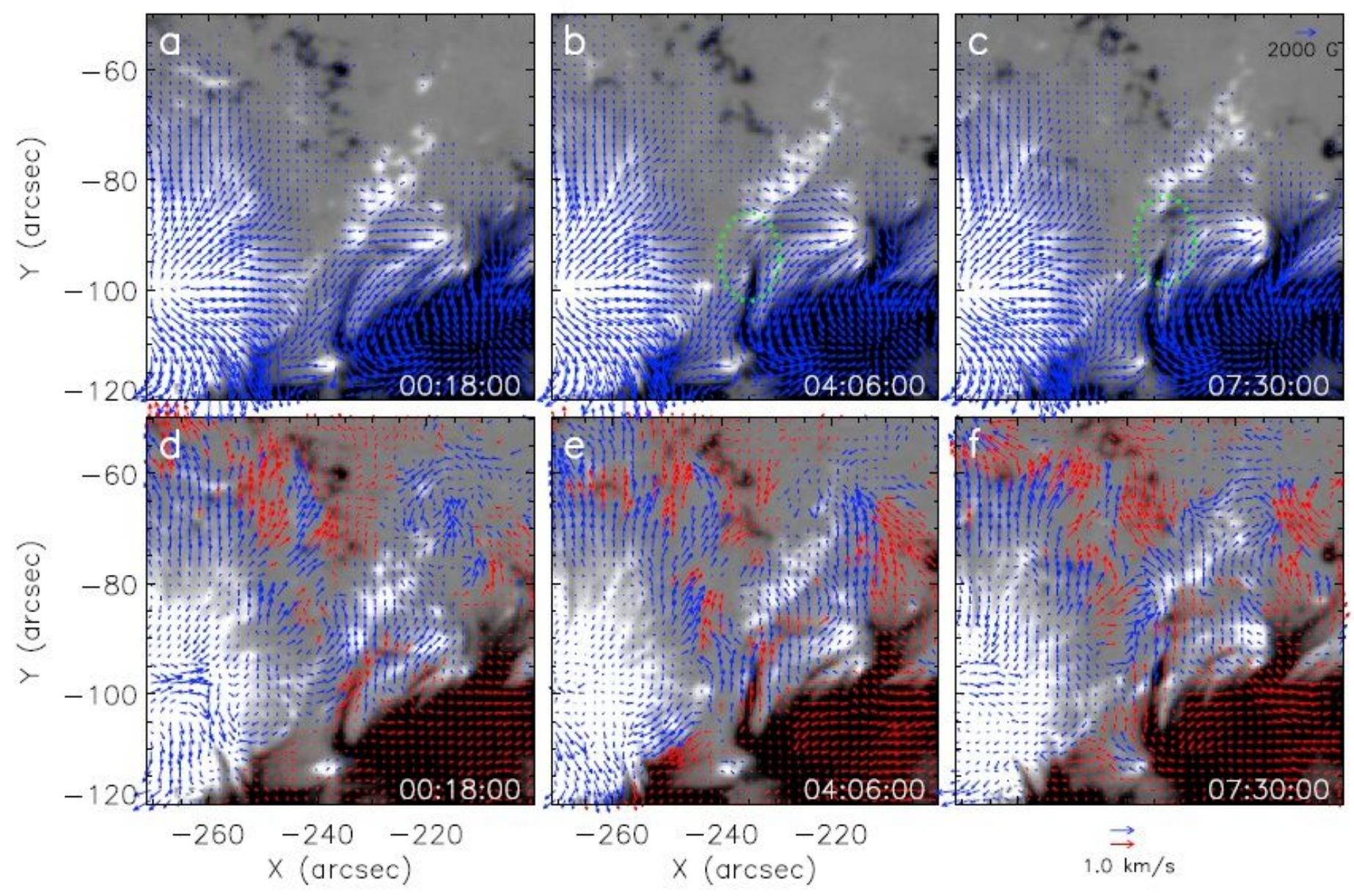

Figure 9

Evolution of the photospheric vector magnetic field observed by SDO/HMI and ow field derived using the DAVE algorithm. (see full caption in Manuscript file)

\section{Supplementary Files}

This is a list of supplementary files associated with this preprint. Click to download.

- SupplementaryMovie1.mp4

- SupplementaryMovie2.mp4

- SupplementaryMovie3.mp4

- SupplementaryMovie4.mp4

- SupplementaryMovie5.mp4 